\title{
Supercritical Nonlinear Vibration of a Fluid-Conveying Pipe Subjected to a Strong External Excitation
}

\author{
Yan-Lei Zhang, ${ }^{1}$ Hui-Rong Feng, ${ }^{2}$ and Li-Qun Chen ${ }^{3,4}$ \\ ${ }^{1}$ College of Engineering, Shanghai Second Polytechnic University, Shanghai 201209, China \\ ${ }^{2}$ College of Transportation and Civil Engineering, Fujian Agriculture and Forestry University, Fuzhou, Fujian 350002, China \\ ${ }^{3}$ Department of Mechanics, Shanghai University, Shanghai 200444, China \\ ${ }^{4}$ Shanghai Institute of Applied Mathematics and Mechanics, Shanghai Key Laboratory of Mechanics in Energy Engineering, \\ Shanghai University, Shanghai 200072, China
}

Correspondence should be addressed to Hui-Rong Feng; 13405948598@163.com

Received 18 June 2015; Accepted 9 September 2015

Academic Editor: Alicia Gonzalez-Buelga

Copyright (c) 2016 Yan-Lei Zhang et al. This is an open access article distributed under the Creative Commons Attribution License, which permits unrestricted use, distribution, and reproduction in any medium, provided the original work is properly cited.

Nonlinear vibration of a fluid-conveying pipe subjected to a transverse external harmonic excitation is investigated in the case with two-to-one internal resonance. The excitation amplitude is in the same magnitude of the transverse displacement. The fluid in the pipes flows in the speed larger than the critical speed so that the straight configuration becomes an unstable equilibrium and two curved configurations bifurcate as stable equilibriums. The motion measured from each of curved equilibrium configurations is governed by a nonlinear integro-partial-differential equation with variable coefficients. The Galerkin method is employed to discretize the governing equation into a gyroscopic system consisting of a set of coupled nonlinear ordinary differential equations. The method of multiple scales is applied to analyze approximately the gyroscopic system. A set of first-order ordinary differential equations governing the modulations of the amplitude and the phase are derived via the method. In the supercritical regime, the subharmonic, superharmonic, and combination resonances are examined in the presence of the $2: 1$ internal resonance. The steadystate responses and their stabilities are determined. The various jump phenomena in the amplitude-frequency response curves are demonstrated. The effects of the viscosity, the excitation amplitude, the nonlinearity, and the flow speed are observed. The analytical results are supported by the numerical integration.

\section{Introduction}

Pipes conveying fluid have been found in many engineering systems such as automobile, aerospace structures, nuclear reactors, boilers, heat exchangers, and steam generators. Due to the widespread applications in many industry fields, the vibration and the stability of fluid-conveying pipes have been extensively investigated, as summarized by Païdoussis $[1,2]$ and Ibrahim $[3,4]$.

The fluid flowing speed plays a crucial role in the dynamics of pipes. Under the critical speed, the straight configuration is the stable equilibrium of the pipe. In such a subcritical regime, Thurman and Mote [5] firstly treated nonlinear vibration and highlighted the significance of the nonlinearity in the case with large speeds. Since then, more and more efforts have been devoted to the study of the nonlinear vibration, including the benchmark paper of Holmes [6] on the subject. It should be motioned that these analyses were extended further for the nonplanar motion of pipes by Ghayesh et al. $[7,8]$. If the fluid speed is larger than the critical speed, the straight pipe configuration becomes unstable and two curved configurations occur as stable equilibriums. Nikolić and Rajković [9] employed the Lyapunov-Schmidt reduction and the singularity theory to analyze stationary bifurcations in fluid-conveying pipes. Plaut [10] applied a shooting method to examine the equilibriums and the vibrations of fluid-conveying pipes. Modarres-Sadeghi and Païdoussis [11] used the finite difference method to investigate supercritical behaviors of extensible pipes. For the case of analysis, Zhang and Chen [12] revealed 2:1 internal resonance of the fluidconveying pipes in the supercritical regime. Sinir [13] showed that many internal resonances might be activated among 
the vibration modes around the same or different buckled configurations. The supercritical problem of pipes with pulsating fluid flow has been studied by Zhang and Chen [14], while few studies have been devoted to the force dynamics of pipes conveying fluid because the external instruction of the governing equation is complicated. All of the abovementioned works have not accounted external excitations.

The pipes are subjected to different environmental actions such as repeated operational startup and shutdown produces. If external excitations cannot be ignored, the pipe motion should be regarded as forced vibration. Chen [15] calculated the response of a cantilevered linear pipe conveying fluids to time-dependent external forces and arbitrary initial conditions. Gulyayev and Tolbatov [16] employed the transfer matrix method to simulate the forced behavior of a pipe containing inner nonhomogeneous flows of a boiling fluid. Seo et al. [17] applied the finite element method to compute the stability and the forced response of a pipe conveying harmonically pulsating fluid. Liang and Wen [18] used the multidimensional Lindstedt-Poincaré method to determine the frequency-amplitude response curves of forced nonlinear vibration of fluid-conveying pipes. It should be remarked that these literatures on pipes with external excitations concern the flow speed in subcritical ranges. To the authors' best knowledge, there is no published literatures on nonlinear forced vibration of the fluid-conveying pipes in the supercritical regime.

To address the lack of researches in the aspect, the present work focuses on nonlinear forced vibration of fluidconveying pipes in the supercritical regime. The amplitude of the external excitation is assumed at the same order of the transverse displacement. In addition to the external resonance, internal resonance is taken into account. Internal resonance with resulting modal interaction among different linear modes is a typical nonlinear phenomenon (Nayfeh and Balachandran [19] Nayfeh [20]). It has been observed in nonlinear vibration of pipes conveying fluids. McDonald and Sri Namachchivaya [21,22] investigated the local and global dynamics of pipes conveying fluid near $0: 1$ internal resonance. $\mathrm{Xu}$ and Yang [23] employed the method of multiple scales to treat the nonlinear modal interaction of the first two modes under external sinusoidal excitation at certain flow velocity. Panda and Kar [24, 25] applied the method of multiple scales to investigate combination and principal parametric resonances in the presence of $3: 1$ internal resonances in pipes conveying pulsating fluid. Ghayesh [26] and Ghayesh et al. [27] highlighted the effects of internal resonance on nonlinear forced dynamics of an axially moving beam, a system similar to a fluid-conveying pipe. All these works on pipes conveying fluids are in the subcritical regime. The published work on internal resonance of fluid-conveying pipes in the supercritical regime is [12-14] while they did not consider external excitations.

The paper is organized as follows. Section 2 presents the equation governing motion measured form a specified curved equilibrium. In Section 3, the frequency and amplitude relationships of subharmonic, superharmonic, and combination and internal resonances occurring simultaneously are derived from the Galerkin truncation and the multiscale

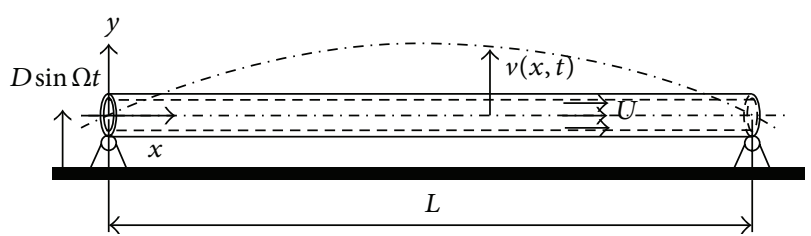

FIGURE 1: A horizontal pipe conveying fluid with external harmonic excitation.

analysis. In Section 4, phenomenon of various jumps is explored in the frequency-response curves. In Section 5, the analytical results are compared with the numerical integration results. Section 6 ends the paper with concluding remarks.

\section{Mathematical Model}

A fluid-conveying pipe at both ends hinged to a transversely moving base is illustrated schematically in Figure 1. The dynamics and stability of tubes conveying fluid are reexamined by means of Euler-Bernoulli beam theory for the tube and a cylindrical shell fluid-mechanical model for the fluid flow. When compared with those equations derived by the cylindrical shell theory (Païdoussis [28-30]), some differences were reported, which were associated with the assumptions. It is shown that this refined theory is necessary for describing adequately the dynamical behaviour of the cross-sectional dimensions of the pipe with respect to its length, although the cylindrical shell theory is quite satisfactory; for long tubes, Euler-Bernoulli beam theory is perfectly adequate.

For the work of the assumptions of pipes conveying fluid the reader is strongly recommended to consult the review articles (Païdoussis [1]). The fluid is assumed to be incompressible, inviscid, and irrotational. The profile of the velocity inside the pipe is constant throughout the pipe. The pipe is modeled as an Euler-Bernoulli beam. Its motion is confined in a plane. Thus the motion of pipe can be described by transverse displacement $v(x, t)$ at neutral axis coordinate $x$ and time $t$. The geometric nonlinearity due to the stretching effect of the midplane of the pipe is accounted. The effect of external damping is neglected here. The gravity effect is also neglected and the pipe is nominally horizontal. If the gravity effect is taken into account, the pipe is with a curved statics equilibrium configuration. However, the analytical procedure is similar if the transverse displacement is measured from the curved equilibrium. With the introduction of the external harmonic excitation, the equation of transverse motion of the pipe is given by (Païdoussis [1])

$$
\begin{aligned}
& (M+m) v_{, t t}+2 M U v_{, t x}+\left[M U^{2}-\bar{P}\right. \\
& \left.-\frac{E A}{2 L} \int_{0}^{L}\left(v_{, x}\right)^{2} \mathrm{~d} x-\frac{E^{*} A}{L} \int_{0}^{L}\left(v_{, x} v_{, t x}\right) \mathrm{d} x\right] v_{, x x} \\
& +E I v_{, x x x x}+E^{*} I v_{, t x x x x}=(M+m) D \Omega^{2} \sin \Omega t
\end{aligned}
$$


with the boundary conditions of simply supports

$$
\begin{aligned}
v(0, t) & =0, \\
v(L, t) & =0 ; \\
v_{, x x}(0, t) & =0, \\
v_{, x x}(L, t) & =0,
\end{aligned}
$$

where $U$ is the fluid velocity, $\bar{P}$ is the externally imposed axial tension, $m$ and $M$ are, respectively, the mass per unit length of pipe and fluid materials, $A$ is the cross-sectional area of the pipe, $L$ is the length, $E I$ is the flexural stiffness of the pipe material, and $E^{*}$ is the viscosity coefficient of internal dissipation of the pipe material which is assumed to be viscoelastic and of the Kelvin-Voigt type. $D$ and $\Omega$ represent external excitation amplitude and frequency, and the comma notation preceding $x$ or $t$ denotes partial derivatives with respect to $x$ or $t$.

Incorporating the following dimensionless quantities:

$$
\begin{aligned}
\eta & =\frac{\nu}{L}, \\
\xi & =\frac{x}{L}, \\
\tau & =\frac{t}{L^{2}} \sqrt{\frac{E I}{M+m}}, \\
u & =L U \sqrt{\frac{M}{E I},} \\
P & =\frac{\bar{P} L^{2}}{E I}, \\
\kappa & =\sqrt{\frac{A L^{2}}{I}}, \\
\alpha & =\frac{E^{*}}{L^{2}} \sqrt{\frac{I}{(M+m) E}}, \\
M_{r} & =\sqrt{\frac{M}{M+m}}, \\
f & =\frac{D}{L}, \\
\omega & =\Omega L^{2} \sqrt{\frac{M+m}{E I}},
\end{aligned}
$$

one can nondimensionalize (1) as

$$
\begin{aligned}
& \eta_{, \tau \tau}+2 M_{r} u \eta_{, \tau \xi} \\
& +\left(u^{2}-P-\frac{\kappa^{2}}{2} \int_{0}^{1}(\eta, \xi)^{2} \mathrm{~d} \xi-\alpha \kappa^{2} \int_{0}^{1}\left(\eta, \xi \eta_{, \tau \xi}\right) \mathrm{d} \xi\right) \\
& \cdot \eta_{, \xi \xi}+\eta_{, \xi \xi \xi \xi}+\alpha \eta_{, \tau \xi \xi \xi \xi}=f \omega^{2} \sin (\omega \tau)
\end{aligned}
$$

and boundary conditions (2) as

$$
\begin{aligned}
\eta(0, \tau) & =0, \\
\eta(1, \tau) & =0 ; \\
\eta_{, \xi \xi}(0, \tau) & =0, \\
\eta_{, \xi \xi}(1, \tau) & =0,
\end{aligned}
$$

where the comma-subscript notation now denotes the partial differentiation with respect to the dimensionless coordinate and time.

The pipe conveying fluid is considered in the supercritical regime. Its pair of curved equilibriums $\widehat{\eta}^{ \pm}(\xi)$ can be derived in a similar way to the case of axially moving beams (Wickert [31]) as

$$
\widehat{\eta}_{k}^{ \pm}(\xi)= \pm \frac{2}{k \pi \kappa} \sqrt{u^{2}-u_{(k)}^{2}} \sin (k \pi \xi), \quad k=1,2,3, \ldots
$$

for $u>u_{(k)}=\sqrt{P+(k \pi)^{2}}$, where $u_{(k)}$ is the critical speed. Over the supercritical range, $u_{(1)}<u<u_{(2)}$, there exist the three solutions $\widehat{\eta}_{0}=0$ (unstable), $\widehat{\eta}_{1}^{-}$(stable), and $\widehat{\eta}_{1}^{+}$(stable). After the transformation of $\eta(\xi, \tau) \leftrightarrow \hat{\eta}_{k}^{ \pm}(\xi)+\eta(\xi, \tau)$, (4) becomes

$$
\begin{aligned}
\eta_{, \tau \tau} & +2 M_{r} u \eta_{, \xi \tau}+u^{2} \eta_{, \xi \xi}+\kappa^{2} \widehat{\eta}_{k}^{ \pm \prime \prime} \int_{0}^{1} \widehat{\eta}_{k}^{ \pm \prime \prime} \eta \mathrm{d} \xi-[P \\
+ & \left.\frac{\kappa^{2}}{2} \int_{0}^{1}\left(\widehat{\eta}_{k}^{ \pm \prime}\right)^{2} \mathrm{~d} \xi\right] \eta_{, \xi \xi}+\eta_{, \xi \xi \xi \xi}+\alpha \eta_{, \tau \xi \xi \xi \xi}=f \omega^{2} \\
& \cdot \sin \omega \tau+\frac{\kappa^{2}}{2}\left(\widehat{\eta}_{k}^{ \pm \prime \prime} \int_{0}^{1} \eta_{, \xi}^{2} \mathrm{~d} \xi-2 \eta_{, \xi \xi} \int_{0}^{1} \widehat{\eta}_{k}^{ \pm \prime \prime} \eta \mathrm{d} \xi\right. \\
& \left.+\eta_{, \xi \xi} \int_{0}^{1} \eta_{, \xi}^{2} \mathrm{~d} \xi\right)+\alpha \kappa^{2} \widehat{\eta}_{k}^{ \pm \prime \prime} \int_{0}^{1} \widehat{\eta}_{k}^{ \pm \prime} \eta_{, \xi \tau} \mathrm{d} \xi \\
& +\alpha \kappa^{2}\left(\eta_{, \xi \xi} \int_{0}^{1} \widehat{\eta}_{k}^{ \pm \prime} \eta_{, \xi \tau} \mathrm{d} \xi+\widehat{\eta}_{k}^{ \pm \prime \prime} \int_{0}^{1} \eta_{, \xi} \eta_{, \xi \tau} \mathrm{d} \xi\right. \\
& \left.+\eta_{, \xi \xi} \int_{0}^{1} \eta_{, \xi} \eta_{, \xi \tau} \mathrm{d} \xi\right),
\end{aligned}
$$

where right superscript ' denotes the differentiation with coordinate. Equation (7) governs the motion measured form the specified curved equilibrium configuration. To express the smallness of the amplitude of pipe motion and the viscosity in the pipe material, they are rescaled as $\eta \leftrightarrow$ $\varepsilon \eta$ and $\alpha \leftrightarrow \varepsilon \alpha$, where the small parameter $\varepsilon$ is a bookkeeping device in the subsequent multiscale analysis. The strong external excitations studied here are with amplitude that can be rescaled as $\varepsilon f$. Substitution of (6) into (7) for $u>$ $u_{(k)}$ leads to a nonlinear integro-partial-differential equation with variable coefficients

$$
\begin{aligned}
\eta_{, \tau \tau}+ & 2 M_{r} u \eta_{, \xi \tau} \\
& +(2 k \pi)^{2}\left(u^{2}-u_{k}^{2}\right) \sin (k \pi \xi) \int_{0}^{1} \eta \sin (k \pi \xi) \mathrm{d} \xi \\
& +(k \pi)^{2} \eta_{, \xi \xi}+\eta_{, \xi \xi \xi \xi}=\varepsilon N(\eta, \tau)+f \omega^{2} \sin \omega \tau,
\end{aligned}
$$


where

$$
\begin{aligned}
& N(\eta, \tau)=-\alpha \eta_{, \tau \xi \xi \xi \xi} \\
& \quad \pm k \pi \kappa \sqrt{u^{2}-u_{k}^{2}}\left[2 \eta_{, \xi \xi} \int_{0}^{1} \eta \sin (k \pi \xi) \mathrm{d} \xi\right. \\
& \left.-\sin (k \pi \xi) \int_{0}^{1} \eta_{, \xi}^{2} \mathrm{~d} \xi\right] \mp 4 k \pi \alpha\left(u^{2}-u_{k}^{2}\right) \sin (k \pi \xi) \\
& \quad \int_{0}^{1} \eta_{, \xi \tau} \cos (k \pi \xi) \mathrm{d} \xi+\frac{\varepsilon \kappa^{2}}{2} \eta_{, \xi \xi}\left(\int_{0}^{1} \eta_{, \xi}^{2} \mathrm{~d} \xi\right. \\
& \left.+2 \varepsilon \alpha \int_{0}^{1} \eta_{, \xi \eta, \xi \tau} \mathrm{d} \xi\right) \\
& \quad \pm \varepsilon \alpha \kappa \sqrt{u^{2}-u_{k}^{2}}\left[2 \eta_{, \xi \xi} \int_{0}^{1} \eta_{, \xi \tau} \cos (k \pi \xi) \mathrm{d} \xi\right. \\
& \left.\quad-2 k \pi \sin (k \pi \xi) \int_{0}^{1} \eta_{, \xi \eta, \xi \tau} \mathrm{d} \xi\right] .
\end{aligned}
$$

\section{Approach of Analysis}

3.1. Truncation via the Galerkin Method. Equation (8) can be cast into a set of ordinary differential equations via the method of Galerkin. Suppose $\eta(\xi, \tau)$ can be approximated by the finite order truncation

$$
\eta(\xi, \tau)=\sum_{r=1}^{n} \phi_{r}(\xi) q_{r}(\tau)
$$

where $\phi_{r}(\xi)$ are eigenfunctions for the free undamped vibrations of a beam satisfying the pinned-pinned boundary conditions (5); namely $\phi_{r}(\xi)=\sqrt{2} \sin (r \pi \xi)$, and $q_{r}(\tau)$ is the $r$ th modal response. In linear vibrations, the low frequency modal responses predominate in the modal expansion because the amplitude of a modal response is proportional to the reciprocal of the square of the frequency. In weakly nonlinear vibrations, it seems reasonable to assume the predomination of the low frequency modal responses. Therefore, truncation order will be chosen as low as possible here to simplify the problem. To account the internal resonance, set $n=2$. The truncation may result in discretization errors and cannot account for all possible internal or external responses.

For greater truncation order, it is necessary to include all of the modes that participate significantly in the response and the larger the number of modes is, the more complicated the dynamics of the system can be. Although the investigation reported in this paper was restricted to a structural system having only two modes, the phenomena described also can occur between any two modes in a multimode system having the appropriate internal resonance due to nonlinear (modal) interactions.

As the flow-rate was increased past a critical value, the position of equilibrium was found to get unstable and bifurcate into the symmetry of steady-state curved equilibrium configuration of a fluid-conveying pipe. The equations governing of $\hat{\eta}_{1}^{+}$positive motion have a form similar to those derived for $\widehat{\eta}_{1}^{-}$negative. Then, in the supercritical regime, the strong forced vibration about a curved equilibrium $\widehat{\eta}_{1}^{+}$ is investigated in the following. Thus, in the case of $k=$ 1 , substituting (10) (with $n=2$ ) into (8), multiplying the resulting equation by weighted function $\phi_{r}(x)$ and integrating the product from 0 to 1 yield a linear gyroscopic system with time-depending forcing terms and small nonlinear terms

$$
\begin{aligned}
\ddot{q}_{1}-G \dot{q}_{2}+k_{11} q_{1}= & f h \sin \omega \tau+\varepsilon \alpha \alpha_{11} \dot{q}_{1}+\varepsilon \kappa \alpha_{12} q_{1}^{2} \\
& +\varepsilon \kappa \alpha_{13} q_{2}^{2}+\varepsilon^{2} \alpha_{14} q_{1}^{3} \\
& +\varepsilon^{2} \alpha_{15} q_{1} q_{2}^{2}+\varepsilon^{2} \alpha_{16} q_{1} \dot{q}_{1} \\
& +\varepsilon^{2} \alpha_{17} q_{2} \dot{q}_{2}+\varepsilon^{3} \alpha_{18} q_{1}^{2} \dot{q}_{1} \\
& +\varepsilon^{3} \alpha_{19} q_{1} q_{2} \dot{q}_{2} \\
\ddot{q}_{2}+G \dot{q}_{1}+k_{21} q_{2}= & \varepsilon \alpha \alpha_{21} \dot{q}_{2}+\varepsilon \kappa \alpha_{22} q_{1} q_{2}+\varepsilon^{2} \alpha_{23} q_{1}^{2} q_{2} \\
& +\varepsilon^{2} \alpha_{24} q_{2}^{3}+\varepsilon^{2} \alpha_{25} q_{2} \dot{q}_{1} \\
& +\varepsilon^{3} \alpha_{26} q_{1} q_{2} \dot{q}_{1}+\varepsilon^{3} \alpha_{27} q_{2}^{2} \dot{q}_{2},
\end{aligned}
$$

where

$$
\begin{aligned}
G & =\frac{16}{3} M_{r} u, \\
k_{11} & =2 \pi^{2}\left(u^{2}-u_{1}^{2}\right), \\
h & =\frac{2 \sqrt{2}}{\pi} \omega^{2}, \\
\alpha_{11} & =-2 \pi^{2}\left(u^{2}-u_{1}^{2}\right)-\pi^{4}, \\
\alpha_{12} & =-\frac{3}{2} \sqrt{2} \pi^{3} \sqrt{u^{2}-u_{1}^{2}}, \\
\alpha_{13} & =-2 \sqrt{2} \pi^{3} \sqrt{u^{2}-u_{1}^{2}}, \\
\alpha_{14} & =-\frac{\kappa^{2}}{2} \pi^{4}, \\
\alpha_{15} & =-2 \kappa^{2} \pi^{4}, \\
\alpha_{16} & =-2 \sqrt{2} \pi^{3} \alpha \kappa \sqrt{u^{2}-u_{1}^{2}}, \\
\alpha_{17} & =-4 \sqrt{2} \pi^{3} \alpha \kappa \sqrt{u^{2}-u_{1}^{2}}, \\
\alpha_{18} & =-\pi^{4} \alpha \kappa^{2}, \\
\alpha_{19} & =-4 \pi^{4} \alpha \kappa^{2}, \\
k_{21} & =12 \pi^{4}, \\
\alpha_{21} & =-16 \pi^{4}, \\
\alpha_{22} & =-4 \sqrt{2} \pi^{3} \sqrt{u^{2}-u_{1}^{2},}
\end{aligned}
$$




$$
\begin{aligned}
& \alpha_{23}=-2 \pi^{4} \kappa^{2}, \\
& \alpha_{24}=-8 \pi^{4} \kappa^{2}, \\
& \alpha_{25}=-4 \sqrt{2} \pi^{3} \alpha \kappa \sqrt{u^{2}-u_{1}^{2}}, \\
& \alpha_{26}=-4 \pi^{4} \alpha \kappa^{2}, \\
& \alpha_{27}=-16 \pi^{4} \alpha \kappa^{2} .
\end{aligned}
$$

The dot represents the differentiation with respect to dimensionless time $\tau$.

3.2. Perturbation via the Method of Multiple Scales. The method of multiple scales (Nayfeh and Mook [32]) can be employed to seek for an approximate solution to (11). The first-order asymptotic expansion of the solutions to (11) can be assumed in the form

$$
\begin{aligned}
& q_{1}=q_{11}\left(T_{0}, T_{1}\right)+\varepsilon q_{12}\left(T_{0}, T_{1}\right)+\cdots \\
& q_{2}=q_{21}\left(T_{0}, T_{1}\right)+\varepsilon q_{22}\left(T_{0}, T_{1}\right)+\cdots,
\end{aligned}
$$

where $T_{0}=\tau$ and $T_{1}=\varepsilon \tau$ are, respectively, the fast and slow time scales. Substituting (13) into (11) and equating coefficients of like powers of $\varepsilon^{0}$ and $\varepsilon^{1}$, one obtains the following.

Order $\varepsilon^{0}$ is as follows:

$$
\begin{aligned}
& D_{0}^{2} q_{11}-G D_{0} q_{21}+k_{11} q_{11}=f h \sin \left(\omega T_{0}\right) \\
& D_{0}^{2} q_{21}+G D_{0} q_{11}+k_{21} q_{21}=0 .
\end{aligned}
$$

Order $\varepsilon^{1}$ is as follows:

$$
\begin{aligned}
D_{0}^{2} q_{12} & -G D_{0} q_{22}+k_{11} q_{12} \\
= & -2 D_{1} D_{0} q_{11}+G D_{1} q_{21}+\alpha \alpha_{11} D_{0} q_{11}+\kappa \alpha_{12} q_{11}^{2} \\
& +\kappa \alpha_{13} q_{21}^{2} \\
D_{0}^{2} q_{22} & +G D_{0} q_{12}+k_{21} q_{22} \\
= & -2 D_{1} D_{0} q_{21}-G D_{1} q_{11}+\alpha \alpha_{21} D_{0} q_{21} \\
& +\kappa \alpha_{22} q_{11} q_{21},
\end{aligned}
$$

where $D_{0}=\partial / \partial T_{0}$ and $D_{1}=\partial / \partial T_{1}$.

Equation (14) defines a 2-degree-of-freedom linear constant-coefficient nonhomogeneous gyroscopic system with a time-depending forcing term. The solution of (14) can be expressed as the general solution to the corresponding homogeneous equation plus a particular solution to the nonhomogeneous equations; namely,

$$
\begin{aligned}
q_{11}= & A_{1}\left(T_{1}\right) \exp \left(i \omega_{1} T_{0}\right)+A_{2}\left(T_{1}\right) \exp \left(i \omega_{2} T_{0}\right) \\
& +f \Lambda_{1} \exp \left(i \omega T_{0}\right)+c c \\
q_{21}= & p_{1} A_{1}\left(T_{1}\right) \exp \left(i \omega_{1} T_{0}\right)+p_{2} A_{2}\left(T_{1}\right) \exp \left(i \omega_{2} T_{0}\right) \\
& +f \Lambda_{2} \exp \left(i \omega T_{0}\right)+c c,
\end{aligned}
$$

where cc stands for complex conjugate of the proceeding terms and

$$
\begin{aligned}
p_{n} & =-\frac{i G \omega_{n}}{k_{21}-\omega_{n}^{2}}=-\frac{i\left(k_{11}-\omega_{n}^{2}\right)}{G \omega_{n}} \\
\Lambda_{1} & =\frac{h\left(\omega^{2}-k_{21}\right)}{2 i\left[G^{2} \omega^{2}-\left(\omega^{2}-k_{11}\right)\left(\omega^{2}-k_{21}\right)\right]}, \\
\Lambda_{2} & =\frac{G h \omega}{2\left[G^{2} \omega^{2}-\left(\omega^{2}-k_{11}\right)\left(\omega^{2}-k_{21}\right)\right]}
\end{aligned}
$$

and the first two natural frequencies of system can be solved from (14) without the forcing term as

$$
\begin{aligned}
\omega_{1,2} & =\frac{\sqrt{2}}{2} \\
\cdot & \sqrt{\left(k_{11}+k_{21}+G^{2}\right) \mp \sqrt{\left(k_{11}-k_{21}+G^{2}\right)^{2}+4 k_{21} G^{2}}} .
\end{aligned}
$$

Substituting (16) into (15) yields

$$
\begin{aligned}
& D_{0}^{2} q_{12}-G D_{0} q_{22}+k_{11} q_{12} \\
& =\left[i \alpha \alpha_{11} \omega_{1} A_{1}-\left(2 i \omega_{1}-G p_{1}\right) D_{1} A_{1}\right] \exp \left(i \omega_{1} T_{0}\right) \\
& +2 \kappa\left[\alpha_{12}+\alpha_{13} p_{1} p_{2}\right] A_{1} A_{2} \exp \left(i\left(\omega_{1}+\omega_{2}\right) T_{0}\right) \\
& +\left[i \alpha \alpha_{11} \omega_{2} A_{2}-\left(2 i \omega_{2}-G p_{2}\right) D_{1} A_{2}\right] \exp \left(i \omega_{2} T_{0}\right) \\
& +2 \kappa\left[\alpha_{12}+\alpha_{13} \bar{p}_{1} p_{2}\right] \bar{A}_{1} A_{2} \exp \left(i\left(\omega_{2}-\omega_{1}\right) T_{0}\right) \\
& +\kappa\left[\alpha_{12}+\alpha_{13} p_{1}^{2}\right] A_{1}^{2} \exp \left(i 2 \omega_{1} T_{0}\right) \\
& +\kappa\left[\alpha_{12}+\alpha_{13} p_{2}^{2}\right] A_{2}^{2} \exp \left(i 2 \omega_{2} T_{0}\right) \\
& +2 \kappa f\left[\alpha_{12} \Lambda_{1}+\alpha_{13} p_{1} \Lambda_{2}\right] A_{1} \exp \left(i\left(\omega+\omega_{1}\right) T_{0}\right) \\
& +2 \kappa f\left[\alpha_{12} \Lambda_{1}+\alpha_{13} p_{2} \Lambda_{2}\right] A_{2} \exp \left(i\left(\omega+\omega_{2}\right) T_{0}\right) \\
& +2 \kappa f\left[\alpha_{12} \Lambda_{1}+\alpha_{13} \bar{p}_{1} \Lambda_{2}\right] \bar{A}_{1} \exp \left(i\left(\omega-\omega_{1}\right) T_{0}\right) \\
& +2 \kappa f\left[\alpha_{12} \Lambda_{1}+\alpha_{13} \bar{p}_{2} \Lambda_{2}\right] \bar{A}_{2} \exp \left(i\left(\omega-\omega_{2}\right) T_{0}\right) \\
& +i f \alpha \omega \alpha_{11} \Lambda_{1} \exp \left(i \omega T_{0}\right)+f^{2} \kappa\left(\alpha_{12} \Lambda_{1}^{2}+\alpha_{13} \Lambda_{2}^{2}\right) \\
& \cdot \exp \left(i 2 \omega T_{0}\right)+c c+\text { NST } \\
& D_{0}^{2} q_{22}+G D_{0} q_{12}+k_{21} q_{22} \\
& =\left[i \alpha \alpha_{21} p_{1} \omega_{1} A_{1}-\left(2 i \omega_{1} p_{1}+G\right) D_{1} A_{1}\right] \\
& \cdot \exp \left(i \omega_{1} T_{0}\right)+\kappa \alpha_{22}\left(p_{1}+p_{2}\right) A_{1} A_{2} \\
& \cdot \exp \left(i\left(\omega_{1}+\omega_{2}\right) T_{0}\right)
\end{aligned}
$$




$$
\begin{aligned}
& +\left[i \alpha \alpha_{21} p_{2} \omega_{2} A_{2}-\left(2 i \omega_{2} p_{2}+G\right) D_{1} A_{2}\right] \\
& \cdot \exp \left(i \omega_{2} T_{0}\right)+\kappa \alpha_{22}\left(\bar{p}_{1}+p_{2}\right) \bar{A}_{1} A_{2} \\
& \cdot \exp \left(i\left(\omega_{2}-\omega_{1}\right) T_{0}\right)+\kappa \alpha_{22} p_{1} A_{1}^{2} \exp \left(i 2 \omega_{1} T_{0}\right) \\
& +\kappa \alpha_{22} p_{2} A_{2}^{2} \exp \left(i 2 \omega_{2} T_{0}\right)+\kappa f \alpha_{22}\left[\Lambda_{1} p_{1}+\Lambda_{2}\right] A_{1} \\
& \cdot \exp \left(i\left(\omega+\omega_{1}\right) T_{0}\right)+\kappa f \alpha_{22}\left[\Lambda_{1} p_{2}+\Lambda_{2}\right] A_{2} \\
& \cdot \exp \left(i\left(\omega+\omega_{2}\right) T_{0}\right)+\kappa f \alpha_{22}\left[\Lambda_{1} \bar{p}_{1}+\Lambda_{2}\right] \bar{A}_{1} \\
& \cdot \exp \left(i\left(\omega-\omega_{1}\right) T_{0}\right)+\kappa f \alpha_{22}\left[\Lambda_{1} \bar{p}_{2}+\Lambda_{2}\right] \bar{A}_{2} \\
& \cdot \exp \left(i\left(\omega-\omega_{2}\right) T_{0}\right)+i f \alpha \omega \alpha_{21} \Lambda_{2} \exp \left(i \omega T_{0}\right) \\
& +f^{2} \kappa \alpha_{22} \Lambda_{1} \Lambda_{2} \exp \left(i 2 \omega T_{0}\right)+\mathrm{cc}+\mathrm{NST},
\end{aligned}
$$

where NST stands for terms that do not produce secular or small-divisor terms and the overbar indicates the complex conjugate. Although the method of multiple scales is an established approach, to authors' best knowledge, there has no treatment on the perturbation of time-dependent gyroscopic systems. In what follows, the case with a twoto-one internal resonance and four fundamental resonances occurring simultaneously is considered.

3.3. Subharmonic Resonance of First Mode: Modulation Equation and Steady-State Responses. Examine the subharmonic resonance of the first mode in the presence of $2: 1$ internal resonance. To describe the nearness of $\omega_{2}$ to $2 \omega_{1}$ and $\omega$ to $2 \omega_{1}$, introduce the detuning parameters $\sigma_{1}$ and $\sigma_{2}$ such that

$$
\begin{gathered}
\omega_{2}=2 \omega_{1}+\varepsilon \sigma_{1}, \\
\omega=2 \omega_{1}+\varepsilon \sigma_{2} .
\end{gathered}
$$

To establish the solvability conditions of gyroscopic system, one assumes a particular solution to (19) in the form

$$
\begin{aligned}
& q_{12}=P_{11} \exp \left(i \omega_{1} T_{0}\right)+P_{12} \exp \left(i \omega_{2} T_{0}\right) \\
& q_{22}=P_{21} \exp \left(i \omega_{1} T_{0}\right)+P_{22} \exp \left(i \omega_{2} T_{0}\right) .
\end{aligned}
$$

Substituting (21) into (19), using (20), and equating the coefficients of $\exp \left(i \omega_{1} T_{0}\right)$ and $\exp \left(i \omega_{2} T_{0}\right)$ on both sides, one obtains

$$
\begin{gathered}
\left(k_{11}-\omega_{n}^{2}\right) P_{1 n}+\left(-i \omega_{n} G\right) P_{2 n}=R_{1 n} \\
\left(i \omega_{n} G\right) P_{1 n}+\left(k_{21}-\omega_{n}^{2}\right) P_{2 n}=R_{2 n}
\end{gathered}
$$

where

$$
\begin{aligned}
R_{11}= & -\left(2 i \omega_{1}-G p_{1}\right) D_{1} A_{1}+i \alpha \alpha_{11} \omega_{1} A_{1} \\
& +2 \kappa\left(\alpha_{12}+\alpha_{13} \bar{p}_{1} p_{2}\right) \bar{A}_{1} A_{2} \exp \left(i \sigma_{1} T_{1}\right) \\
& +2 f \kappa\left(\alpha_{12} \Lambda_{1}+\alpha_{13} \Lambda_{2} \bar{p}_{1}\right) \bar{A}_{1} \exp \left(i \sigma_{2} T_{1}\right)
\end{aligned}
$$

$$
\begin{aligned}
R_{12}= & -\left(2 i \omega_{2}-G p_{2}\right) D_{1} A_{2}+i \alpha \alpha_{11} \omega_{2} A_{2} \\
& +\kappa\left(\alpha_{12}+\alpha_{13} p_{1}^{2}\right) A_{1}^{2} \exp \left(-i \sigma_{1} T_{1}\right) \\
& +i f \alpha\left(2 \omega_{1}+\varepsilon \sigma_{2}\right) \alpha_{11} \Lambda_{1} \exp \left(i\left(\sigma_{2}-\sigma_{1}\right) T_{1}\right) \\
R_{21}= & -\left(2 i \omega_{1} p_{1}+G\right) D_{1} A_{1}+i \alpha \alpha_{21} \omega_{1} p_{1} A_{1} \\
& +\kappa \alpha_{22}\left(\bar{p}_{1}+p_{2}\right) \bar{A}_{1} A_{2} \exp \left(i \sigma_{1} T_{1}\right) \\
& +f \kappa \alpha_{22}\left(\Lambda_{2}+\bar{p}_{1} \Lambda_{1}\right) \bar{A}_{1} \exp \left(i \sigma_{2} T_{1}\right) \\
R_{22}= & -\left(2 i \omega_{2} p_{2}+G\right) D_{1} A_{2}+i \alpha \alpha_{21} \omega_{2} p_{2} A_{2} \\
& +\kappa \alpha_{22} p_{1} A_{1}^{2} \exp \left(-i \sigma_{1} T_{1}\right) \\
& +i f \alpha\left(2 \omega_{1}+\varepsilon \sigma_{2}\right) \alpha_{21} \Lambda_{2} \exp \left(i\left(\sigma_{2}-\sigma_{1}\right) T_{1}\right) .
\end{aligned}
$$

The natural frequencies, given by (18), make the determinant of the coefficient matrix (22) vanish. The existence of solutions to (22) demands

$$
\left|\begin{array}{cc}
R_{1 n} & -i \omega_{n} G \\
R_{2 n} & k_{21}-\omega_{n}^{2}
\end{array}\right|=0
$$

Thus, the solvability condition of subharmonic resonance of the first mode in presence of $2: 1$ internal resonance is derived from (24) as

$$
R_{1 n}=-\bar{p}_{n} R_{2 n}
$$

Substituting (23) into (25) and rearranging the resulting equation lead to

$$
\begin{aligned}
D_{1} A_{1}= & \alpha \Gamma_{11} A_{1}+\kappa \Gamma_{12} \bar{A}_{1} A_{2} \exp \left(i \sigma_{1} T_{1}\right) \\
& +f \kappa \Gamma_{13} \bar{A}_{1} \exp \left(i \sigma_{2} T_{1}\right) \\
D_{1} A_{2}= & \alpha \Gamma_{21} A_{2}+\kappa \Gamma_{22} A_{1}^{2} \exp \left(-i \sigma_{1} T_{1}\right) \\
& +f \alpha \Gamma_{23} \exp \left(i\left(\sigma_{2}-\sigma_{1}\right) T_{1}\right),
\end{aligned}
$$

where

$$
\begin{aligned}
\Gamma_{11} & =\frac{i \alpha_{11} \omega_{1}+i \alpha_{21} \omega_{1} p_{1} \bar{p}_{1}}{\bar{p}_{1}\left(2 i \omega_{1} p_{1}+G\right)+\left(2 i \omega_{1}-G p_{1}\right)}, \\
\Gamma_{12} & =\frac{2 \alpha_{12}+2 \alpha_{13} \bar{p}_{1} p_{2}+\alpha_{22}\left(\bar{p}_{1}+p_{2}\right) \bar{p}_{1}}{\bar{p}_{1}\left(2 i \omega_{1} p_{1}+G\right)+\left(2 i \omega_{1}-G p_{1}\right)}, \\
\Gamma_{13} & =\frac{2 \alpha_{12} \Lambda_{1}+2 \alpha_{13} \bar{p}_{1} \Lambda_{2}+\alpha_{22}\left(\Lambda_{2}+\bar{p}_{1} \Lambda_{1}\right) \bar{p}_{1}}{\bar{p}_{1}\left(2 i \omega_{1} p_{1}+G\right)+\left(2 i \omega_{1}-G p_{1}\right)},
\end{aligned}
$$




$$
\begin{aligned}
\Gamma_{21} & =\frac{i \alpha_{11} \omega_{2}+i \alpha_{21} \omega_{2} p_{2} \bar{p}_{2}}{\bar{p}_{2}\left(2 i \omega_{2} p_{2}+G\right)+\left(2 i \omega_{2}-G p_{2}\right)}, \\
\Gamma_{22} & =\frac{\alpha_{12}+\alpha_{13} p_{1}^{2}+\alpha_{22} p_{1} \bar{p}_{2}}{\bar{p}_{2}\left(2 i \omega_{2} p_{2}+G\right)+\left(2 i \omega_{2}-G p_{2}\right)}, \\
\Gamma_{23} & =\frac{i\left(2 \omega_{1}+\varepsilon \sigma_{2}\right) \alpha_{11} \Lambda_{1}+i\left(2 \omega_{1}+\varepsilon \sigma_{2}\right) \alpha_{21} \Lambda_{2} \bar{p}_{2}}{\bar{p}_{2}\left(2 i \omega_{2} p_{2}+G\right)+\left(2 i \omega_{2}-G p_{2}\right)} .
\end{aligned}
$$

To express the amplitude of motion conveniently, the polar transformation for the complex amplitude $A_{n}$ can be introduced as

$$
A_{n}\left(T_{1}\right)=\frac{1}{2} a_{n}\left(T_{1}\right) \exp \left[i \theta_{n}\left(T_{1}\right)\right],
$$

where $a_{n}$ and $\theta_{n}$ are the amplitude and the phase that are real valued functions. Substituting (28) into (26) and separating the resulting equation into real and imaginary parts yield the modulation equation:

$$
\begin{aligned}
\frac{\mathrm{d} a_{1}}{\mathrm{~d} T_{1}}= & \alpha \Gamma_{11}^{R} a_{1}+\frac{\kappa}{2}\left(\Gamma_{12}^{R} \cos \gamma_{1}-\Gamma_{12}^{I} \sin \gamma_{1}\right) a_{1} a_{2} \\
& +f \kappa\left(\Gamma_{13}^{R} \cos \gamma_{2}-\Gamma_{13}^{I} \sin \gamma_{2}\right) a_{1} \\
\frac{\mathrm{d} a_{2}}{\mathrm{~d} T_{1}}= & \alpha \Gamma_{21}^{R} a_{2}+\frac{\kappa}{2}\left(\Gamma_{22}^{R} \cos \gamma_{1}+\Gamma_{22}^{I} \sin \gamma_{1}\right) a_{1}^{2} \\
& +2 f \alpha\left[\Gamma_{23}^{R} \cos \left(\gamma_{2}-\gamma_{1}\right)-\Gamma_{23}^{I} \sin \left(\gamma_{2}-\gamma_{1}\right)\right] \\
a_{2} \frac{\mathrm{d} \gamma_{1}}{\mathrm{~d} T_{1}}= & \left.\sigma_{1}+\alpha \Gamma_{21}^{I}\right) a_{2} \\
& -\frac{\kappa}{2}\left(\Gamma_{22}^{R} \sin \gamma_{1}-\Gamma_{22}^{I} \cos \gamma_{1}\right) a_{1}^{2} \\
& +2 f \alpha\left[\Gamma_{23}^{R} \sin \left(\gamma_{2}-\gamma_{1}\right)+\Gamma_{23}^{I} \cos \left(\gamma_{2}-\gamma_{1}\right)\right] \\
& -2 \alpha \Gamma_{11}^{I} a_{2}-\kappa\left(\Gamma_{12}^{R} \sin \gamma_{1}+\Gamma_{12}^{I} \cos \gamma_{1}\right) a_{2}^{2} \\
& -2 f \kappa\left(\Gamma_{13}^{R} \sin \gamma_{2}+\Gamma_{13}^{I} \cos \gamma_{2}\right) a_{2} \\
\frac{\mathrm{d} \gamma_{2}}{\mathrm{~d} T_{1}}= & \sigma_{2}-2 \alpha \Gamma_{11}^{I}-\kappa\left(\Gamma_{12}^{R} \sin \gamma_{1}+\Gamma_{12}^{I} \cos \gamma_{1}\right) a_{2} \\
& -2 f \kappa\left(\Gamma_{13}^{R} \sin \gamma_{2}+\Gamma_{13}^{I} \cos \gamma_{2}\right),
\end{aligned}
$$

where $\gamma_{1}=\theta_{2}-2 \theta_{1}+T_{1} \sigma_{1}$ and $\gamma_{2}=-2 \theta_{1}+T_{1} \sigma_{2}$. The right superscripts $R$ and $I$ denote, respectively, the real and the imaginary parts of each coefficient.

For the steady-state response, $a_{n}$ and $\gamma_{n}$ are constants. Therefore the left-hand side of (29) should be zero. The resulting equations have two possible solutions. In the case termed as the single-mode solution $\left(a_{1}=0, a_{2} \neq 0\right)$, one has trivial linear solutions:

$$
\begin{aligned}
& a_{1}=a_{1 l}=0 \\
& a_{2}=a_{2 l}=\frac{2 f \alpha\left|\Gamma_{23}\right|}{\sqrt{\left(\alpha \Gamma_{21}^{R}\right)^{2}+\left(\sigma_{2}-\sigma_{1}-\alpha \Gamma_{21}^{I}\right)^{2}}} .
\end{aligned}
$$

In the second case, there are coupled two-mode solutions $\left(a_{1} \neq 0, a_{2} \neq 0\right)$ that can be numerically solved.

To determine the stability of the nontrivial coupled twomode solutions, one derives the disturbance equation of (29) as

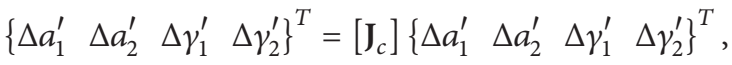

where right superscript $T$ denotes transpose and $\left[\mathbf{J}_{c}\right]$ is the Jacobian matrix calculated at the coupled two-mode solution. The eigenvalues of $\left[\mathbf{J}_{c}\right]$ determine the stability of steadystate responses corresponding to the two-mode solutions. The solution is stable if and only if the real parts of eigenvalues are less than or equal to zero.

\subsection{Superharmonic Resonance of Second Mode: Modulation} Equation and Steady-State Responses. The superharmonic resonance of second mode and modal interactions may occur simultaneously. In this case, to describe quantitatively the nearness of these resonances, one introduces the detuning parameters $\sigma_{1}$ and $\sigma_{2}$ such that

$$
\begin{aligned}
& \omega_{2}=2 \omega_{1}+\varepsilon \sigma_{1}, \\
& 2 \omega=\omega_{2}+\varepsilon \sigma_{2} .
\end{aligned}
$$

In order to avoid secular terms, the solvability conditions of second superharmonic resonance in presence of internal resonance can be derived in the similar way in Section 3.3. Substituting particular solution (21) into (19), considering (32), and equating the coefficients of $\exp \left(i \omega_{1} T_{0}\right)$ and $\exp \left(i \omega_{2} T_{0}\right)$ on both sides, one obtains

$$
\begin{gathered}
\left(k_{11}-\omega_{n}^{2}\right) P_{1 n}+\left(-i \omega_{n} G\right) P_{2 n}=S_{1 n} \\
\left(i \omega_{n} G\right) P_{1 n}+\left(k_{21}-\omega_{n}^{2}\right) P_{2 n}=S_{2 n}
\end{gathered}
$$

where

$$
\begin{aligned}
S_{11}= & -\left(2 i \omega_{1}-G p_{1}\right) D_{1} A_{1}+i \alpha \alpha_{11} \omega_{1} A_{1} \\
& +2 \kappa\left(\alpha_{12}+\alpha_{13} \bar{p}_{1} p_{2}\right) \bar{A}_{1} A_{2} \exp \left(i \sigma_{1} T_{1}\right) \\
S_{12}= & -\left(2 i \omega_{2}-G p_{2}\right) D_{1} A_{2}+i \alpha \alpha_{11} \omega_{2} A_{2} \\
& +\kappa\left(\alpha_{12}+\alpha_{13} p_{1}^{2}\right) A_{1}^{2} \exp \left(-i \sigma_{1} T_{1}\right) \\
& +f^{2} \kappa\left(\alpha_{12} \Lambda_{1}^{2}+\alpha_{13} \Lambda_{2}^{2}\right) \exp \left(i \sigma_{2} T_{1}\right) \\
S_{21}= & \left(2 i \omega_{1} p_{1}+G\right) D_{1} A_{1}+i \alpha \alpha_{21} \omega_{1} p_{1} A_{1} \\
& +\kappa \alpha_{22}\left(\bar{p}_{1}+p_{2}\right) \bar{A}_{1} A_{2} \exp \left(i \sigma_{1} T_{1}\right) \\
S_{22}= & \left(2 i \omega_{2} p_{2}+G\right) D_{1} A_{2}+i \alpha \alpha_{21} \omega_{2} p_{2} A_{2} \\
& +\kappa \alpha_{22} p_{1} A_{1}^{2} \exp \left(-i \sigma_{1} T_{1}\right) \\
& +f^{2} \kappa \alpha_{22} \Lambda_{1} \Lambda_{2} \exp \left(i \sigma_{2} T_{1}\right) .
\end{aligned}
$$

Thus, (33) has solutions only when the solvability condition

$$
S_{1 n}=-\bar{p}_{n} S_{2 n}
$$


holds. Substituting (34) into (35) and rearranging terms in the resulting equation lead to the modulation equations:

$$
\begin{aligned}
D_{1} A_{1}= & \alpha \Gamma_{11} A_{1}+\kappa \Gamma_{12} \bar{A}_{1} A_{2} \exp \left(i \sigma_{1} T_{1}\right) \\
D_{1} A_{2}= & \alpha \Gamma_{21} A_{2}+\kappa \Gamma_{22} A_{1}^{2} \exp \left(-i \sigma_{1} T_{1}\right) \\
& +f^{2} \kappa \Gamma_{25} \exp \left(i \sigma_{2} T_{1}\right),
\end{aligned}
$$

where the $\Gamma_{11}, \Gamma_{12}, \Gamma_{21}$, and $\Gamma_{21}$ terms are defined by (27) in Section 3.3 and

$$
\Gamma_{25}=\frac{\alpha_{12} \Lambda_{1}^{2}+\alpha_{13} \Lambda_{2}^{2}+\alpha_{22} \Lambda_{1} \Lambda_{2} \bar{p}_{2}}{\bar{p}_{2}\left(2 i \omega_{2} p_{2}+G\right)+\left(2 i \omega_{2}-G p_{2}\right)} .
$$

Substituting (28) into (36) and separating the resulting equation into real and imaginary parts, one obtains the polar equations:

$$
\begin{aligned}
\frac{\mathrm{d} a_{1}}{\mathrm{~d} T_{1}}= & \alpha \Gamma_{11}^{R} a_{1}+\frac{\kappa}{2}\left(\Gamma_{12}^{R} \cos \gamma_{1}-\Gamma_{12}^{I} \sin \gamma_{1}\right) a_{1} a_{2} \\
\frac{\mathrm{d} a_{2}}{\mathrm{~d} T_{1}}= & \alpha \Gamma_{21}^{R} a_{2}+\frac{\kappa}{2}\left(\Gamma_{22}^{R} \cos \gamma_{1}+\Gamma_{22}^{I} \sin \gamma_{1}\right) a_{1}^{2} \\
& +2 f^{2} \kappa\left(\Gamma_{25}^{R} \cos \gamma_{2}-\Gamma_{25}^{I} \sin \gamma_{2}\right) \\
a_{2} \frac{\mathrm{d} \gamma_{1}}{\mathrm{~d} T_{1}}= & \left(\sigma_{1}+\alpha \Gamma_{21}^{I}\right) a_{2} \\
& -\frac{\kappa}{2}\left(\Gamma_{22}^{R} \sin \gamma_{1}-\Gamma_{22}^{I} \cos \gamma_{1}\right) a_{1}^{2} \\
& +2 f^{2} \kappa\left(\Gamma_{25}^{R} \sin \gamma_{2}+\Gamma_{25}^{I} \cos \gamma_{2}\right) \\
& -2 \alpha \Gamma_{11}^{I} a_{2}-\kappa\left(\Gamma_{12}^{R} \sin \gamma_{1}+\Gamma_{12}^{I} \cos \gamma_{1}\right) a_{2}^{2} \\
a_{2} \frac{\mathrm{d} \gamma_{2}}{\mathrm{~d} T_{1}}= & \left(\sigma_{2}-\alpha \Gamma_{21}^{I}\right) a_{2} \\
& +\frac{\kappa}{2}\left(\Gamma_{22}^{R} \sin \gamma_{1}-\Gamma_{22}^{I} \cos \gamma_{1}\right) a_{1}^{2} \\
& -2 f^{2} \kappa\left(\Gamma_{25}^{R} \sin \gamma_{2}+\Gamma_{25}^{I} \cos \gamma_{2}\right)
\end{aligned}
$$

where $\gamma_{1}=\theta_{2}-2 \theta_{1}+T_{1} \sigma_{1}$ and $\gamma_{2}=-\theta_{2}+T_{1} \sigma_{2}$.

The steady-state response is with constants $a_{n}$ and $\gamma_{n}$ defined by (38) with zero left-hand side. There are two types of steady-state responses. The single-mode solution $\left(a_{1}=0\right.$, $\left.a_{2} \neq 0\right)$ is

$$
\begin{aligned}
& a_{1}=a_{1 l}=0 \\
& a_{2}=a_{2 l}=\frac{2 f^{2} \kappa\left|\Gamma_{25}\right|}{\sqrt{\left(\alpha \Gamma_{21}^{R}\right)^{2}+\left(\sigma_{2}-\alpha \Gamma_{21}^{I}\right)^{2}}} .
\end{aligned}
$$

The two-mode solutions $\left(a_{1} \neq 0, a_{2} \neq 0\right)$ can be numerically solved. Their stabilities can be determined by the eigenvalues of the Jacobian matrix of first-order differential equations (38). If the real parts of the eigenvalues are all negative then the steady-state solution is stable.
3.5. Summation Resonance: Modulation Equation and SteadyState Responses. In this case, the frequency relations for the $2: 1$ internal resonance and the combination resonance of the sum type are

$$
\begin{aligned}
& \omega_{2}=2 \omega_{1}+\varepsilon \sigma_{1}, \\
& \omega=\omega_{1}+\omega_{2}+\varepsilon \sigma_{2},
\end{aligned}
$$

where $\sigma_{1}$ and $\sigma_{2}$ are the detuning parameters.

Following the similar arguments as in Section 3.3, the solvability conditions can be determined for the summation resonance in presence of internal resonance. Substituting particular solution (21) into (19), using conditions (40), and equating the coefficients of $\exp \left(i \omega_{1} T_{0}\right)$ and $\exp \left(i \omega_{2} T_{0}\right)$ on both sides lead to

$$
\begin{gathered}
\left(k_{11}-\omega_{n}^{2}\right) P_{1 n}+\left(-i \omega_{n} G\right) P_{2 n}=M_{1 n} \\
\left(i \omega_{n} G\right) P_{1 n}+\left(k_{21}-\omega_{n}^{2}\right) P_{2 n}=M_{2 n},
\end{gathered}
$$

where

$$
\begin{aligned}
M_{11}= & -\left(2 i \omega_{1}-G p_{1}\right) D_{1} A_{1}+i \alpha \alpha_{11} \omega_{1} A_{1} \\
& +2 \kappa\left(\alpha_{12}+\alpha_{13} \bar{p}_{1} p_{2}\right) \bar{A}_{1} A_{2} \exp \left(i \sigma_{1} T_{1}\right) \\
& +2 f \kappa\left(\alpha_{12} \Lambda_{1}+\alpha_{13} \Lambda_{2} \bar{p}_{2}\right) \bar{A}_{2} \exp \left(i \sigma_{2} T_{1}\right) \\
M_{12}= & -\left(2 i \omega_{2}-G p_{2}\right) D_{1} A_{2}+i \alpha \alpha_{11} \omega_{2} A_{2} \\
& +\kappa\left(\alpha_{12}+\alpha_{13} p_{1}^{2}\right) A_{1}^{2} \exp \left(-i \sigma_{1} T_{1}\right) \\
& +2 f \kappa\left(\alpha_{12} \Lambda_{1}+\alpha_{13} \Lambda_{2} \bar{p}_{1}\right) \bar{A}_{1} \exp \left(i \sigma_{2} T_{1}\right) \\
M_{21}= & -\left(2 i \omega_{1} p_{1}+G\right) D_{1} A_{1}+i \alpha \alpha_{21} \omega_{1} p_{1} A_{1} \\
& +\kappa \alpha_{22}\left(\bar{p}_{1}+p_{2}\right) \bar{A}_{1} A_{2} \exp \left(i \sigma_{1} T_{1}\right) \\
& +f \kappa \alpha_{22}\left(\Lambda_{2}+\Lambda_{1} \bar{p}_{2}\right) \bar{A}_{2} \exp \left(i \sigma_{2} T_{1}\right) \\
M_{22}= & \left(2 i \omega_{2} p_{2}+G\right) D_{1} A_{2}+i \alpha \alpha_{21} \omega_{2} p_{2} A_{2} \\
& +\kappa \alpha_{22} p_{1} A_{1}^{2} \exp \left(-i \sigma_{1} T_{1}\right) \\
& +f \kappa \alpha_{22}\left(\Lambda_{2}+\Lambda \bar{p}_{1}\right) \bar{A}_{1} \exp \left(i \sigma_{2} T_{1}\right) .
\end{aligned}
$$

Then the solvability condition of the simultaneous summation and internal resonances are

$$
M_{1 n}=-\bar{p}_{n} M_{2 n}
$$

Thus, substituting (42) into (43) and rearranging the resulting equation yield the modulation equations:

$$
\begin{aligned}
D_{1} A_{1}= & \alpha \Gamma_{11} A_{1}+\kappa \Gamma_{12} \bar{A}_{1} A_{2} \exp \left(i \sigma_{1} T_{1}\right) \\
& +f \kappa \Gamma_{16} \bar{A}_{2} \exp \left(i \sigma_{2} T_{1}\right) \\
D_{1} A_{2}= & \alpha \Gamma_{21} A_{2}+\kappa \Gamma_{22} A_{1}^{2} \exp \left(-i \sigma_{1} T_{1}\right) \\
& +f \kappa \Gamma_{26} \bar{A}_{1} \exp \left(i \sigma_{2} T_{1}\right),
\end{aligned}
$$


where the $\Gamma_{11}, \Gamma_{12}, \Gamma_{21}$, and $\Gamma_{21}$ terms are defined by (27) and

$$
\begin{aligned}
\Gamma_{16} & =\frac{2 \alpha_{12} \Lambda_{1}+2 \alpha_{13} \Lambda_{2} \bar{p}_{2}+\alpha_{22}\left(\Lambda_{2}+\Lambda_{1} \bar{p}_{2}\right) \bar{p}_{1}}{\bar{p}_{1}\left(2 i \omega_{1} p_{1}+G\right)+\left(2 i \omega_{1}-G p_{1}\right)} \\
\Gamma_{26} & =\frac{2 \alpha_{12} \Lambda_{1}+2 \alpha_{13} \Lambda_{2} \bar{p}_{1}+\alpha_{22}\left(\Lambda_{2}+\Lambda_{1} \bar{p}_{1}\right) \bar{p}_{2}}{\bar{p}_{2}\left(2 i \omega_{2} p_{2}+G\right)+\left(2 i \omega_{2}-G p_{2}\right)} .
\end{aligned}
$$

Substituting the polar expression (28) into (44) and separating the resulting equation into real and imaginary parts yield

$$
\begin{aligned}
& \frac{\mathrm{d} a_{1}}{\mathrm{~d} T_{1}}=\alpha \Gamma_{11}^{R} a_{1}+\frac{\kappa}{2}\left(\Gamma_{12}^{R} \cos \gamma_{1}-\Gamma_{12}^{I} \sin \gamma_{1}\right) a_{1} a_{2} \\
& +f_{\kappa}\left(\Gamma_{16}^{R} \cos \gamma_{2}-\Gamma_{16}^{I} \sin \gamma_{2}\right) a_{2} \\
& \frac{\mathrm{d} a_{2}}{\mathrm{~d} T_{1}}=\alpha \Gamma_{21}^{R} a_{2}+\frac{\kappa}{2}\left(\Gamma_{22}^{R} \cos \gamma_{1}+\Gamma_{22}^{I} \sin \gamma_{1}\right) a_{1}^{2} \\
& +f \kappa\left(\Gamma_{26}^{R} \cos \gamma_{2}-\Gamma_{26}^{I} \sin \gamma_{2}\right) a_{1} \\
& a_{1} a_{2} \frac{\mathrm{d} \gamma_{1}}{\mathrm{~d} T_{1}}=\left(\sigma_{1}+\alpha \Gamma_{21}^{I}\right) a_{1} a_{2} \\
& -\frac{\kappa}{2}\left(\Gamma_{22}^{R} \sin \gamma_{1}-\Gamma_{22}^{I} \cos \gamma_{1}\right) a_{1}^{3} \\
& +f \kappa\left(\Gamma_{26}^{R} \sin \gamma_{2}+\Gamma_{26}^{I} \cos \gamma_{2}\right) a_{1}^{2} \\
& -2 \alpha \Gamma_{11}^{I} a_{1} a_{2} \\
& -\kappa\left(\Gamma_{12}^{R} \sin \gamma_{1}+\Gamma_{12}^{I} \cos \gamma_{1}\right) a_{1} a_{2}^{2} \\
& -2 f \kappa\left(\Gamma_{16}^{R} \sin \gamma_{2}+\Gamma_{16}^{I} \cos \gamma_{2}\right) a_{2}^{2} \\
& a_{1} a_{2} \frac{\mathrm{d} \gamma_{2}}{\mathrm{~d} T_{1}}=\left(\sigma_{2}-\alpha \Gamma_{11}^{I}\right) a_{1} a_{2} \\
& -\frac{\kappa}{2}\left(\Gamma_{12}^{R} \sin \gamma_{1}+\Gamma_{12}^{I} \cos \gamma_{1}\right) a_{1} a_{2}^{2} \\
& -f \kappa\left(\Gamma_{16}^{R} \sin \gamma_{2}+\Gamma_{16}^{I} \cos \gamma_{2}\right) a_{2}^{2} \\
& -\alpha \Gamma_{21}^{I} a_{1} a_{2} \\
& +\frac{\kappa}{2}\left(\Gamma_{22}^{R} \sin \gamma_{1}-\Gamma_{22}^{I} \cos \gamma_{1}\right) a_{1}^{3} \\
& -f \kappa\left(\Gamma_{26}^{R} \sin \gamma_{2}+\Gamma_{26}^{I} \cos \gamma_{2}\right) a_{1}^{2},
\end{aligned}
$$

where $\gamma_{1}=\theta_{2}-2 \theta_{1}+T_{1} \sigma_{1}$ and $\gamma_{2}=-\theta_{1}-\theta_{2}+T_{1} \sigma_{2}$.

For the steady-state response, the constants $a_{n}$ and $\gamma_{n}$ are defined by (46) with zero left-hand side. There are two possible types of steady-state response. The first is the trivial zero solution $a_{1}=0, a_{2}=0$ corresponding to the equilibrium. The second is the nontrivial coupled solutions $a_{1} \neq 0, a_{2} \neq 0$ that can be numerically solved. The stability of the nontrivial steady-state response can be analyzed by examining the eigenvalues of the Jacobian matrix of (46).

3.6. Difference Resonance: Modulation Equation and SteadyState Responses. In this case, the combination resonance of the differential type with $2: 1$ internal resonance is considered. Use the detuning parameters $\sigma_{1}$ and $\sigma_{2}$ to describe the nearness of $\omega_{2}$ to $2 \omega_{1}$ and $\omega$ to $\omega_{2}-\omega_{1}$, respectively. Thus, the frequency relations are given by

$$
\begin{aligned}
\omega_{2} & =2 \omega_{1}+\varepsilon \sigma_{1}, \\
\omega & =\omega_{2}-\omega_{1}+\varepsilon \sigma_{2},
\end{aligned}
$$

where $\sigma_{1}$ and $\sigma_{2}$ are the detuning parameters.

Thus, substituting particular solutions (21) into (19), using (47), and equating the coefficients of $\exp \left(i \omega_{1} T_{0}\right)$ and $\exp \left(i \omega_{2} T_{0}\right)$ on both sides yield

$$
\begin{gathered}
\left(k_{11}-\omega_{n}^{2}\right) P_{1 n}+\left(-i \omega_{n} G\right) P_{2 n}=N_{1 n} \\
\left(i \omega_{n} G\right) P_{1 n}+\left(k_{21}-\omega_{n}^{2}\right) P_{2 n}=N_{2 n},
\end{gathered}
$$

where

$$
\begin{aligned}
& N_{11}=-\left(2 i \omega_{1}-G p_{1}\right) D_{1} A_{1}+i \alpha \alpha_{11} \omega_{1} A_{1} \\
& \quad+2 \kappa\left(\alpha_{12}+\alpha_{13} \bar{p}_{1} p_{2}\right) \bar{A}_{1} A_{2} \exp \left(i \sigma_{1} T_{1}\right) \\
& \quad+2 f \kappa\left(\alpha_{12} \bar{\Lambda}_{1}+\alpha_{13} \bar{\Lambda}_{2} p_{2}\right) A_{2} \exp \left(-i \sigma_{2} T_{1}\right) \\
& \quad+i f \alpha\left[\omega_{1}+\varepsilon\left(\sigma_{1}+\sigma_{2}\right)\right] \alpha_{11} \Lambda_{1} \exp \left(i\left(\sigma_{1}+\sigma_{2}\right) T_{1}\right) \\
& N_{12}=-\left(2 i \omega_{2}-G p_{2}\right) D_{1} A_{2}+i \alpha \alpha_{11} \omega_{2} A_{2} \\
& \quad+\kappa\left(\alpha_{12}+\alpha_{13} p_{1}^{2}\right) A_{1}^{2} \exp \left(-i \sigma_{1} T_{1}\right) \\
& \quad+2 f \kappa\left(\alpha_{12} \Lambda_{1}+\alpha_{13} \Lambda_{2} p_{1}\right) A_{1} \exp \left(i \sigma_{2} T_{1}\right) \\
& N_{21}=-\left(2 i \omega_{1} p_{1}+G\right) D_{1} A_{1}+i \alpha \alpha_{21} \omega_{1} p_{1} A_{1} \\
& \quad+\kappa \alpha_{22}\left(\bar{p}_{1}+p_{2}\right) \bar{A}_{1} A_{2} \exp \left(i \sigma_{1} T_{1}\right) \\
& \quad+f \kappa \alpha_{22}\left(\bar{\Lambda}_{2}+\bar{\Lambda}_{1} p_{2}\right) A_{2} \exp \left(-i \sigma_{2} T_{1}\right) \\
& \quad+i f \alpha\left[\omega_{1}+\varepsilon\left(\sigma_{1}+\sigma_{2}\right)\right] \alpha_{21} \Lambda_{2} \exp \left(i\left(\sigma_{1}+\sigma_{2}\right) T_{1}\right) \\
& \quad+f \kappa \alpha_{22}\left(\Lambda_{2}+\Lambda_{1} p_{1}\right) A_{1} \exp \left(i \sigma_{2} T_{1}\right) \\
& N_{22}=-\left(2 i \omega_{2} p_{2}+G\right) D_{1} A_{2}+i \alpha \alpha_{21} \omega_{2} p_{2} A_{2} \\
& +\kappa \alpha_{22} p_{1} A_{1}^{2} \exp \left(-i \sigma_{1} T_{1}\right)
\end{aligned}
$$

According to similar lines as in previous case, the solvability condition of the differences resonance in the presence of internal resonance case is

$$
N_{1 n}=-\bar{p}_{n} N_{2 n}
$$

Substituting (49) into (50) and rearranging the resulting equation give

$$
\begin{aligned}
D_{1} A_{1}= & \alpha \Gamma_{11} A_{1}+\kappa \Gamma_{12} \bar{A}_{1} A_{2} \exp \left(i \sigma_{1} T_{1}\right) \\
& +f \kappa \Gamma_{17} A_{2} \exp \left(-i \sigma_{2} T_{1}\right) \\
& +f \alpha \Gamma_{18} \exp \left(i\left(\sigma_{1}+\sigma_{2}\right) T_{1}\right) \\
D_{1} A_{2}= & \alpha \Gamma_{21} A_{2}+\kappa \Gamma_{22} A_{1}^{2} \exp \left(-i \sigma_{1} T_{1}\right) \\
& +f \kappa \Gamma_{27} A_{1} \exp \left(i \sigma_{2} T_{1}\right),
\end{aligned}
$$


where the $\Gamma_{11}, \Gamma_{12}, \Gamma_{21}$, and $\Gamma_{21}$ term are defined by (27) and

$$
\begin{aligned}
& \Gamma_{17}=\frac{2 \alpha_{12} \bar{\Lambda}_{1}+2 \alpha_{13} \bar{\Lambda}_{2} p_{2}+\alpha_{22}\left(\bar{\Lambda}_{2}+\bar{\Lambda}_{1} p_{2}\right) \bar{p}_{1}}{\bar{p}_{1}\left(2 i \omega_{1} p_{1}+G\right)+\left(2 i \omega_{1}-G p_{1}\right)} \\
& \Gamma_{18} \\
& =\frac{i\left[\omega_{1}+\varepsilon\left(\sigma_{1}+\sigma_{2}\right)\right] \alpha_{11} \Lambda_{1}+i\left[\omega_{1}+\varepsilon\left(\sigma_{1}+\sigma_{2}\right)\right] \alpha_{21} \Lambda_{2} \bar{p}_{1}}{\bar{p}_{1}\left(2 i \omega_{1} p_{1}+G\right)+\left(2 i \omega_{1}-G p_{1}\right)} \\
& \Gamma_{27}=\frac{2 \alpha_{12} \Lambda_{1}+2 \alpha_{13} \Lambda_{2} p_{1}+\alpha_{22}\left(\Lambda_{2}+\Lambda_{1} p_{1}\right) \bar{p}_{2}}{\bar{p}_{2}\left(2 i \omega_{2} p_{2}+G\right)+\left(2 i \omega_{2}-G p_{2}\right)} .
\end{aligned}
$$

Substituting (28) into (51) and separating the resulting equation into real and imaginary parts yield

$$
\begin{aligned}
& \frac{\mathrm{d} a_{1}}{\mathrm{~d} T_{1}} \\
& =\alpha \Gamma_{11}^{R} a_{1}+\frac{\kappa}{2}\left(\Gamma_{12}^{R} \cos \gamma_{1}-\Gamma_{12}^{I} \sin \gamma_{1}\right) a_{1} a_{2} \\
& \quad+f \kappa\left(\Gamma_{17}^{R} \cos \gamma_{2}-\Gamma_{17}^{I} \sin \gamma_{2}\right) a_{2} \\
& \quad+2 f \alpha\left[\Gamma_{18}^{R} \cos \left(\gamma_{1}-\gamma_{2}\right)-\Gamma_{18}^{I} \sin \left(\gamma_{1}-\gamma_{2}\right)\right]
\end{aligned}
$$

$\frac{\mathrm{d} a_{2}}{\mathrm{~d} T_{1}}$

$$
\begin{aligned}
& =\alpha \Gamma_{21}^{R} a_{2}+\frac{\kappa}{2}\left(\Gamma_{22}^{R} \cos \gamma_{1}+\Gamma_{22}^{I} \sin \gamma_{1}\right) a_{1}^{2} \\
& +f \kappa\left(\Gamma_{27}^{R} \cos \gamma_{2}+\Gamma_{27}^{I} \sin \gamma_{2}\right) a_{1} \\
& a_{1} a_{2} \frac{\mathrm{d} \gamma_{1}}{\mathrm{~d} T_{1}} \\
& =\left(\sigma_{1}+\alpha \Gamma_{21}^{I}\right) a_{1} a_{2}-\frac{\kappa}{2}\left(\Gamma_{22}^{R} \sin \gamma_{1}-\Gamma_{22}^{I} \cos \gamma_{1}\right) a_{1}^{3} \\
& -f \kappa\left(\Gamma_{27}^{R} \sin \gamma_{2}-\Gamma_{27}^{I} \cos \gamma_{2}\right) a_{1}^{2}-2 \alpha \Gamma_{11}^{I} a_{1} a_{2} \\
& -\kappa\left(\Gamma_{12}^{R} \sin \gamma_{1}+\Gamma_{12}^{I} \cos \gamma_{1}\right) a_{1} a_{2}^{2} \\
& -2 f \kappa\left(\Gamma_{17}^{R} \sin \gamma_{2}+\Gamma_{17}^{I} \cos \gamma_{2}\right) a_{2}^{2} \\
& -4 f \alpha\left[\Gamma_{18}^{R} \sin \left(\gamma_{1}-\gamma_{2}\right)+\Gamma_{18}^{I} \cos \left(\gamma_{1}-\gamma_{2}\right)\right] a_{2} \\
& a_{1} a_{2} \frac{\mathrm{d} \gamma_{2}}{\mathrm{~d} T_{1}} \\
& =-\left(\sigma_{2}-\alpha \Gamma_{21}^{I}\right) a_{1} a_{2} \\
& -\frac{\kappa}{2}\left(\Gamma_{22}^{R} \sin \gamma_{1}-\Gamma_{22}^{I} \cos \gamma_{1}\right) a_{1}^{3} \\
& -f \kappa\left(\Gamma_{27}^{R} \sin \gamma_{2}-\Gamma_{27}^{I} \cos \gamma_{2}\right) a_{1}^{2}-\alpha \Gamma_{11}^{I} a_{1} a_{2} \\
& -\frac{\kappa}{2}\left(\Gamma_{12}^{R} \sin \gamma_{1}+\Gamma_{12}^{I} \cos \gamma_{1}\right) a_{1} a_{2}^{2} \\
& -f \kappa\left(\Gamma_{17}^{R} \sin \gamma_{2}+\Gamma_{17}^{I} \cos \gamma_{2}\right) a_{2}^{2} \\
& -2 f \alpha\left[\Gamma_{18}^{R} \sin \left(\gamma_{1}-\gamma_{2}\right)+\Gamma_{18}^{I} \cos \left(\gamma_{1}-\gamma_{2}\right)\right] a_{2},
\end{aligned}
$$

where $\gamma_{1}=\theta_{2}-2 \theta_{1}+T_{1} \sigma_{1}$ and $\gamma_{2}=\theta_{2}-\theta_{1}-T_{1} \sigma_{2}$.
For the steady-state responses, $a_{n}$ and $\gamma_{n}$ are constants. In this case, there is neither the single-mode solution nor the trivial zero solution. The coupled solutions $\left(a_{1} \neq 0\right.$, $a_{2} \neq 0$ ) can be numerically solved, and their stabilities can be determined by the eigenvalues of the Jacobian matrix of (53).

\section{Numerical Demonstrations of Analytical Results}

This section presents numerical demonstrations of the analytical results. A control approach by changing an objective parameter at each time was developed using the NewtonSimpson's iterative method for solution of the nonlinear modulation equations. The vibration amplitude band structure of the dynamics response was calculated to determine the gap frequency range. Through careful numerical simulations and stability analysis, it is shown that most fine details of the dynamical structure, found in the reduced equations, have their corresponding counterpart in the original coupled twodegree-of-freedom system.

Novel jumping phenomena can be observed in the supercritical regime. The natural frequencies for the first and the second modes are evaluated as functions of the fluid velocity $u$ for specific values of other system parameters. Taking the fluid-pipe mass ration $M_{r}=0.447$ and initial tension parameter $P=-5$, one finds that if the nondimensional flow velocity $u=5.02704$, the first natural frequency $\left(\omega_{2}=37.0432\right)$ is equal to two times of the first one $\left(\omega_{1}=18.5216\right)$. In this case, there may be $2: 1$ internal resonance. The corresponding internal detuning parameter $\sigma_{1}=0$. The frequency detuning of the strong external excitations is taken as the control parameters. In the calculations, the book-keeping parameter $\varepsilon$ is chosen as 0.01 . In the following figures, the solid lines represent stable steady-state responses and the dashed lines represent the unstable ones.

4.1. First Subharmonic Resonance: Steady-State Responses and Their Stabilities. In the analysis of the pipe-fluid system subjected to subharmonic resonance of first mode (i.e., $\omega \approx$ $2 \omega_{1}$ ) in presence of $2: 1$ internal resonance (with $M_{r}=0.447$, $P=-5, \omega_{1}=18.5216$, and $\left.\omega_{2}=37.0432\right)$, a set of typical values of system parameters are taken as $u=5.02704$, $\alpha=0.001, f=0.001$, and $\kappa=4$. To reveal the changing trend with the parameters, different valves of $u, \alpha, f$, and $\kappa$ are also assigned. The nonlinear steady-state responses along with their stability and bifurcations are examined via utilizing the reduced equations representing the modulations in the system response.

Figure 2 demonstrates the amplitude-frequency response curves of the first subharmonic resonance for different viscosity coefficients ( $\alpha=0.001,0.002,0.003$, and 0.005$)$. The response curves in this case have the single-mode and the coupled two-mode steady-state responses. As shown in Figure 2(a), in the first mode, double-peak jumping exists for the small enough viscosity coefficient, and the amplitude decreases with the viscosity coefficient. For the second mode, as show in Figure 2(b), there is a coupled two-mode response with a straight-up peak separating the two-peak jumping 


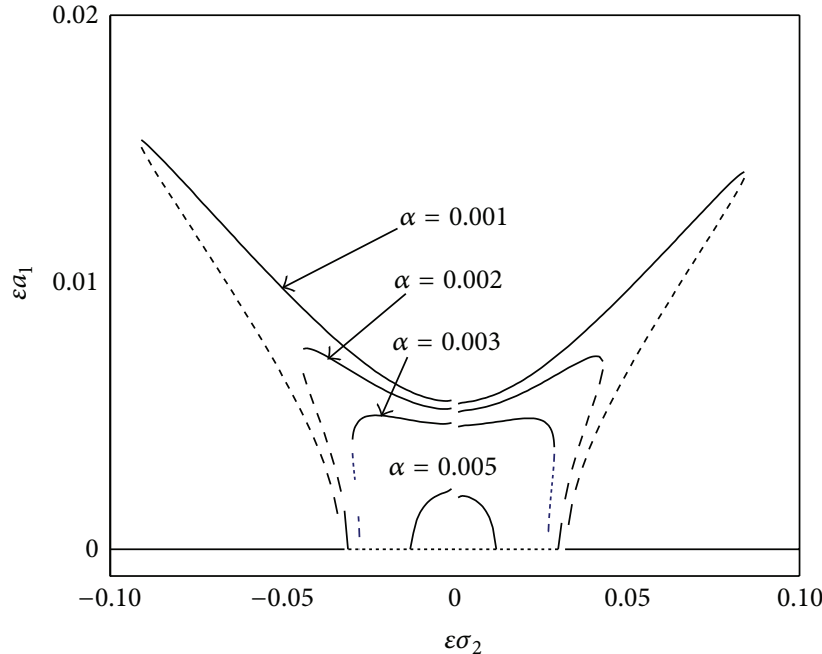

(a)

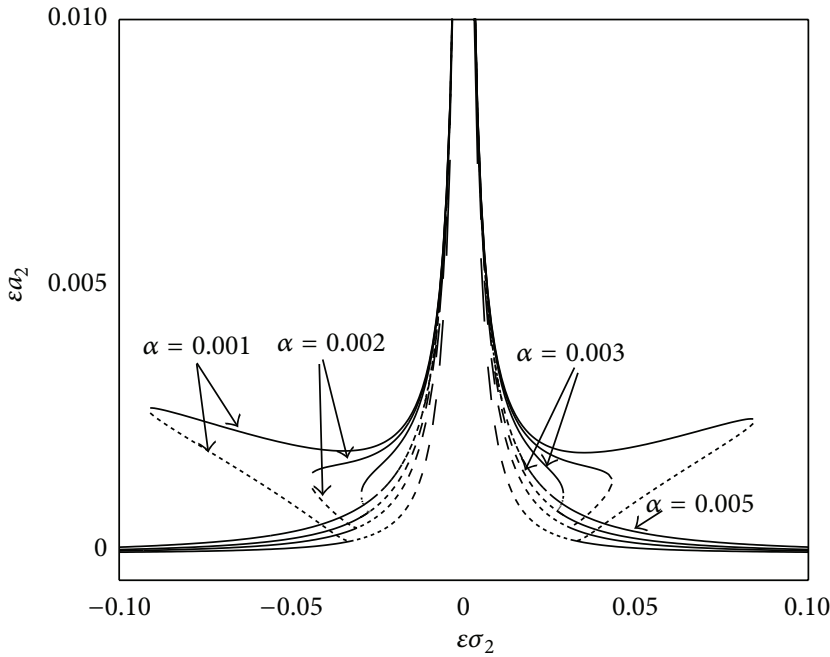

(b)

FIGURE 2: Amplitude-frequency curves of the first subharmonic resonance for different viscosity coefficients: (a) the first mode and (b) the second mode.

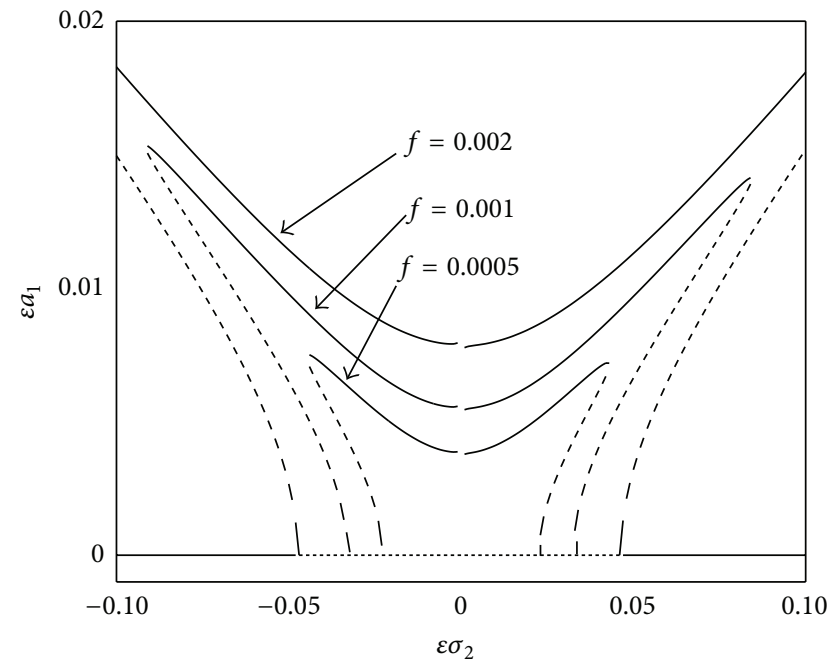

(a)

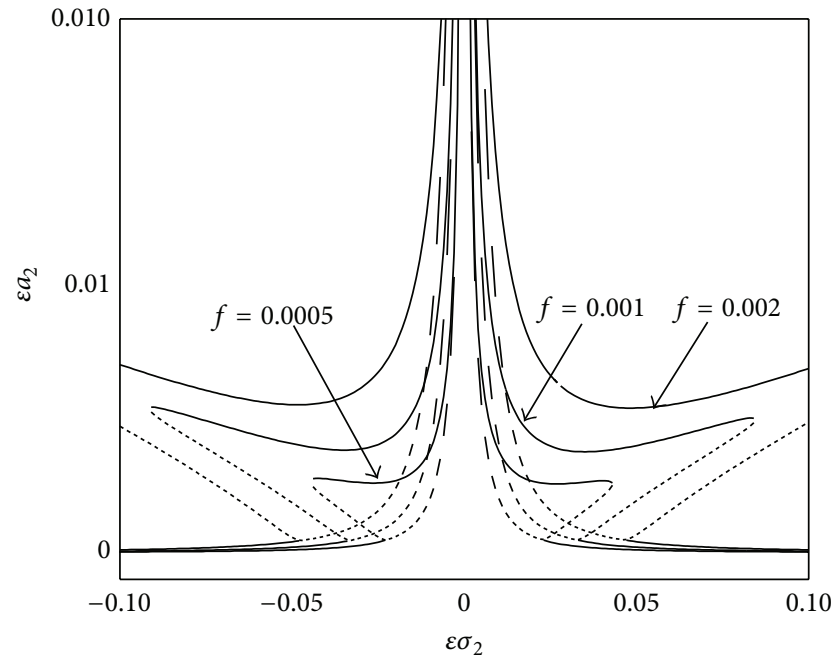

(b)

FIGURE 3: Amplitude-frequency curves of the first subharmonic resonance for different external excitation amplitudes: (a) the first mode and (b) the second mode.

and an unstable single-mode response. For the coupled twomode steady-state response, the amplitude of the first-mode response is much larger than that of the second-mode one.

Figure 3 illustrates the amplitude-frequency response curves of the first subharmonic resonance for different external excitation amplitudes ( $f=0.0005,0.001$, and 0.002$)$. As shown in Figure 3 the amplitudes of both the single-mode response and the coupled two-mode response increase with the increasing amplitudes of external excitation.
Figure 4 shows the amplitude-frequency response curves of the first subharmonic resonance for different nonlinearity coefficients $(\kappa=2,4$, and 6). The amplitudes of the coupled two-mode response increase with the increasing nonlinearity coefficients, while the amplitudes of the singlemode response are independent of the coefficient.

Figure 5 depicts the amplitude-frequency response curves of the first subharmonic resonance for different flow speeds $(u=5.025,5.02704$, and 5.030). The coupled 


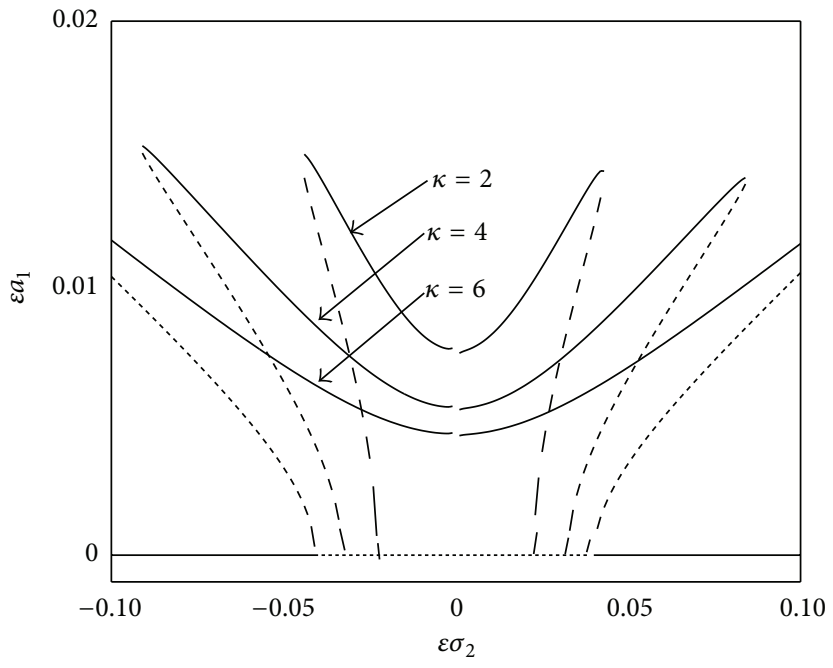

(a)

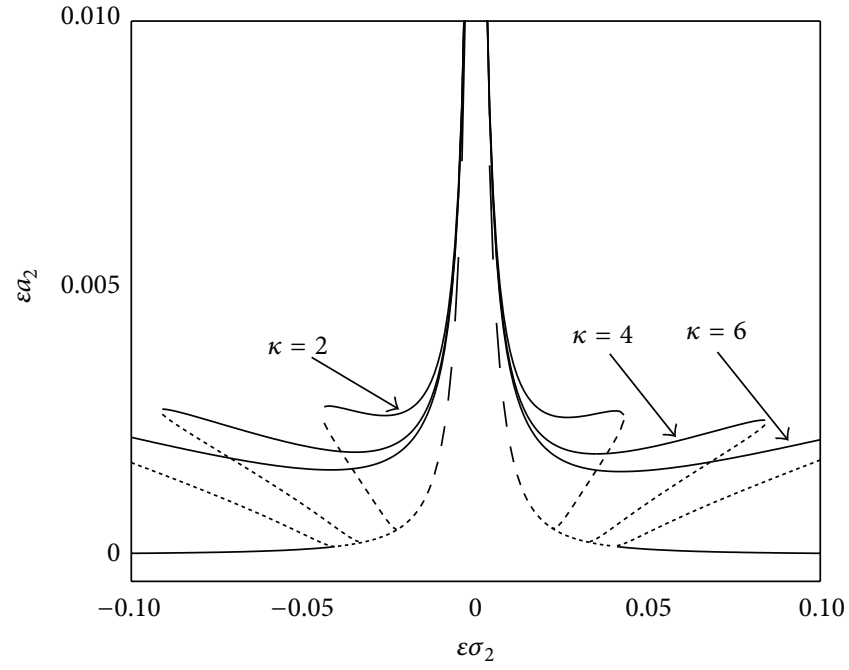

(b)

Figure 4: Amplitude-frequency curves of first subharmonic resonance for different nonlinearity coefficients: (a) the first mode and (b) the second mode.

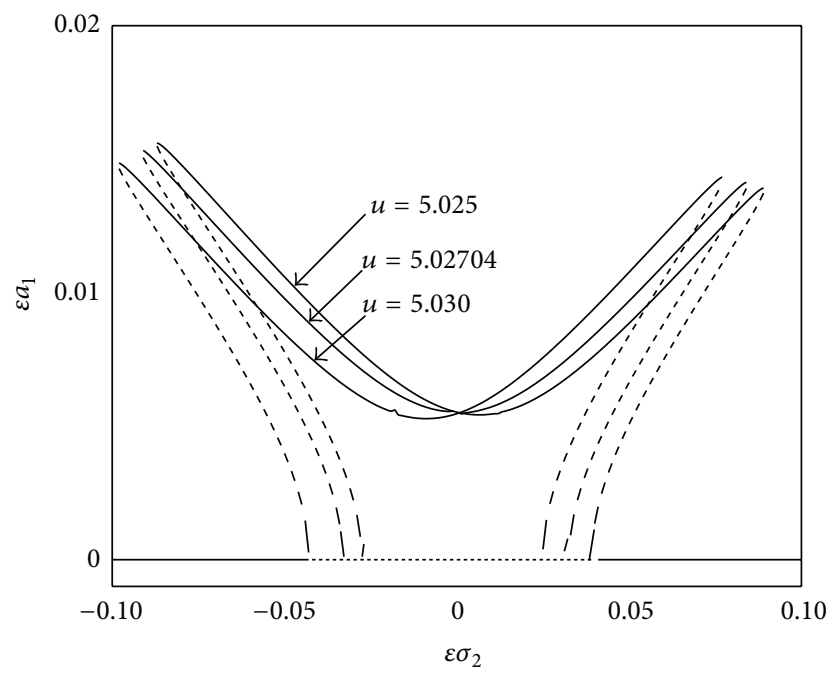

(a)

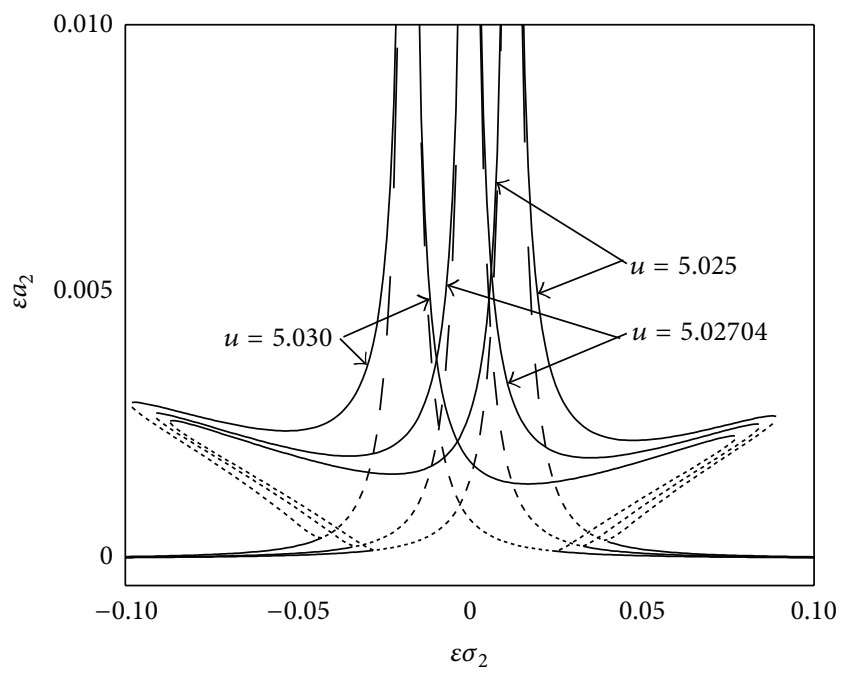

(b)

Figure 5: Amplitude-frequency curves of first subharmonic resonance for different flow speeds: (a) the first mode and (b) the second mode.

two-mode responses become unsymmetrical in the near but not exact internal resonance $\left(\sigma_{1} \neq 0\right)$.

In the first subharmonic resonance with the $2: 1$ internal resonance, the pipe vibration becomes severe abruptly when the excitation frequency is slightly larger or smaller than 2 times of the first natural frequency.

4.2. Second Superharmonic Resonance: Steady-State Responses and Their Stabilities. To investigate the superharmonic resonance of the second mode (i.e., $2 \omega \approx \omega_{2}$ ) in presence of $2: 1$ internal resonance, the parameters are chosen as $M_{r}=0.447$, $P=-5, u=5.02704, \alpha=0.001, f=0.001$, and $\kappa=4$ unless other values are assigned.

Figure 6 illustrates the amplitude-frequency response curves of the second superharmonic resonance for different viscosity coefficients ( $\alpha=0.001,0.002,0.003$, and 0.005$)$. As shown in Figure 6(a), in the first mode, jumping only exists for small viscosity coefficients, and two types of jumping are separated by a high no-jumping peak in the amplitude-frequency curves. As shown in Figure 6(b), in 


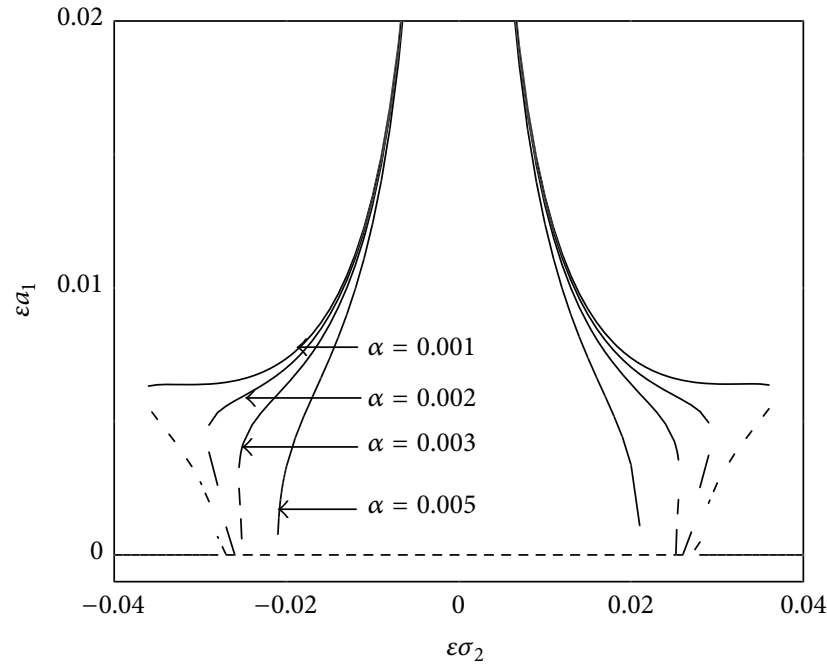

(a)

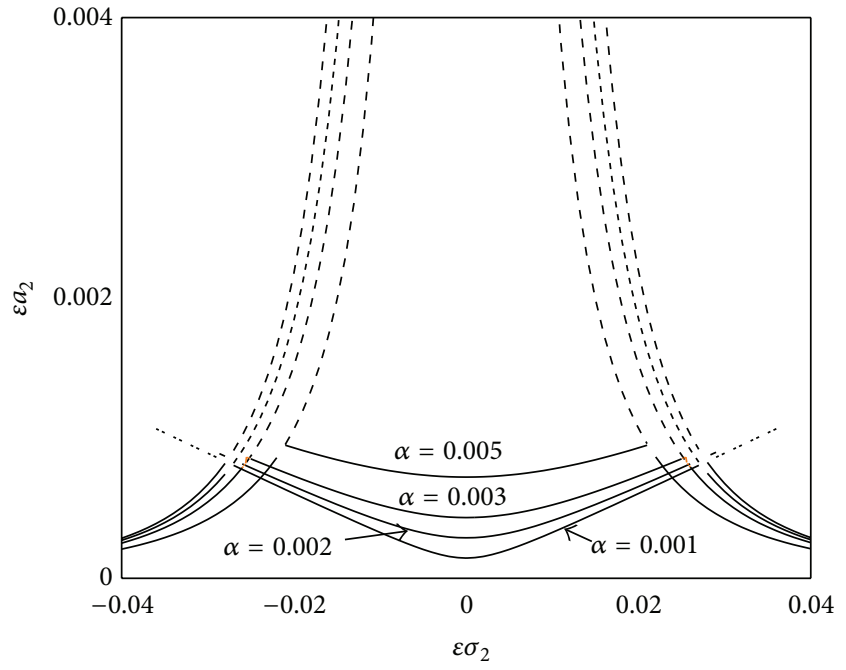

(b)

FIGURE 6: Amplitude-frequency curves of the second superharmonic resonance for different viscosity coefficients: (a) the first mode and (b) the second mode.

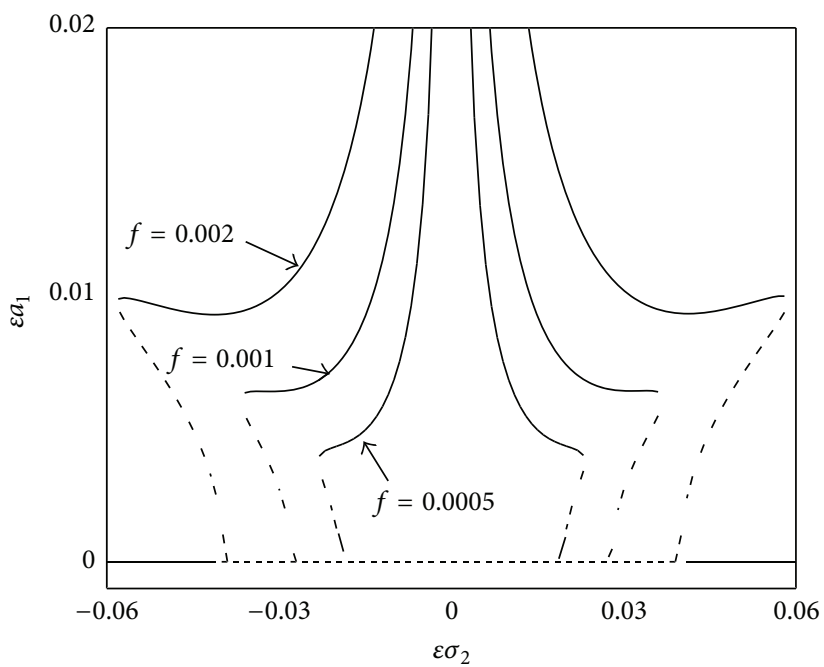

(a)

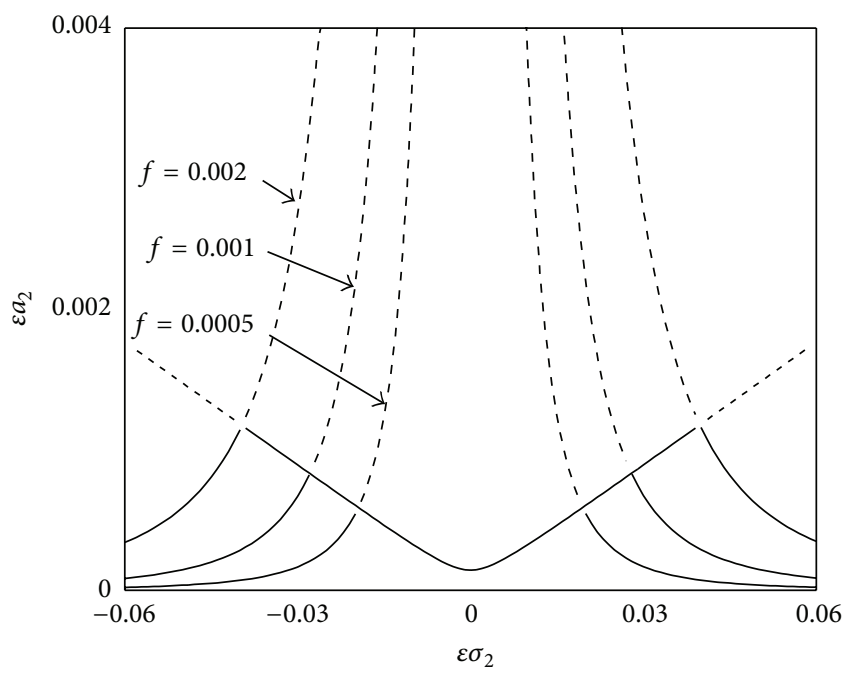

(b)

FIGURE 7: Amplitude-frequency curves of the second superharmonic resonance for different external excitation amplitudes: (a) the first mode and (b) the second mode.

the second mode, there are exceptional small coupled twomode responses and unstable single-mode responses. The amplitude of the first-mode response is much larger than that of the second-mode one.

Figure 7 demonstrates the amplitude-frequency response curves of the second superharmonic resonance for different external excitation amplitudes $(f=0.0005,0.001$, and 0.002). As shown in Figure 7, the amplitudes of both the coupled two-mode response in the first mode and the unstable single-mode response in the second mode increase with the increasing amplitudes of external excitation, while the amplitudes of coupled two-mode response in the second mode are independent of the excitation amplitudes.

Figure 8 shows the amplitude-frequency response curve of the second superharmonic resonance for different nonlinearity coefficients $(\kappa=2,4$, and 6). As shown in Figure 8, the amplitudes of both the single-mode response and the coupled two-mode response decrease with the nonlinearity coefficients.

Figure 9 depicts the amplitude-frequency response curves of the second superharmonic resonant for different flow speeds $(u=5.025,5.02704$, and 5.030). The coupled 


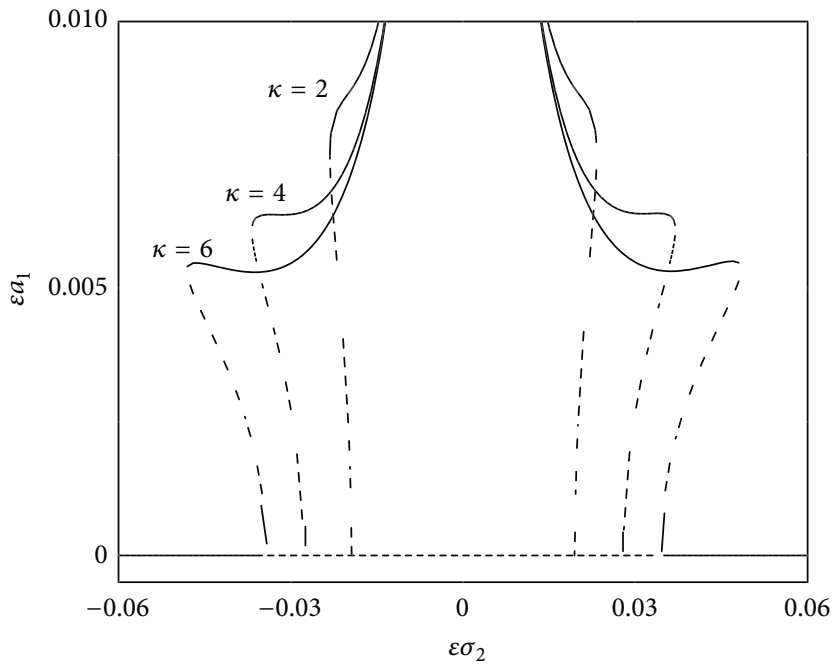

(a)

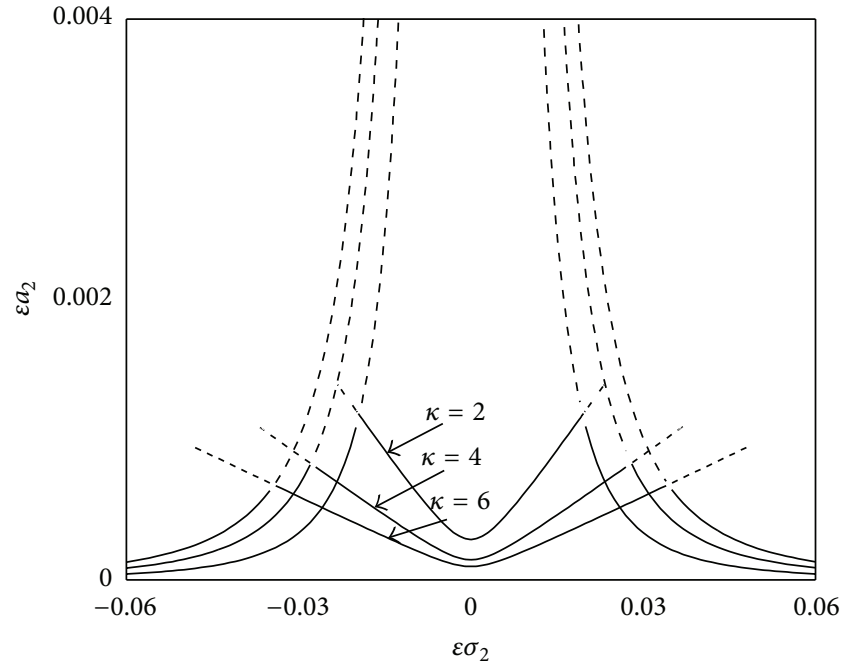

(b)

Figure 8: Amplitude-frequency curves of the second superharmonic resonance for different nonlinearity coefficients: (a) the first mode and (b) the second mode.

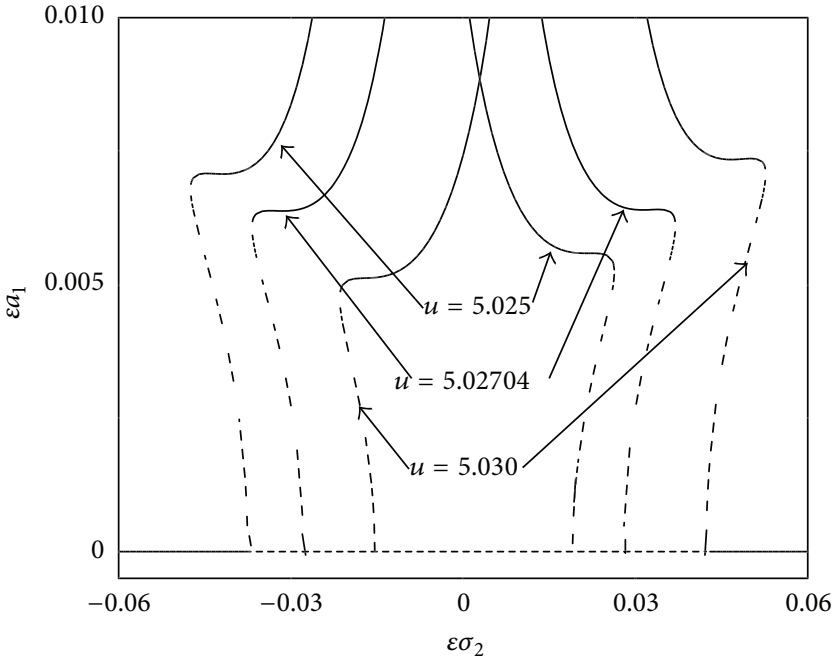

(a)

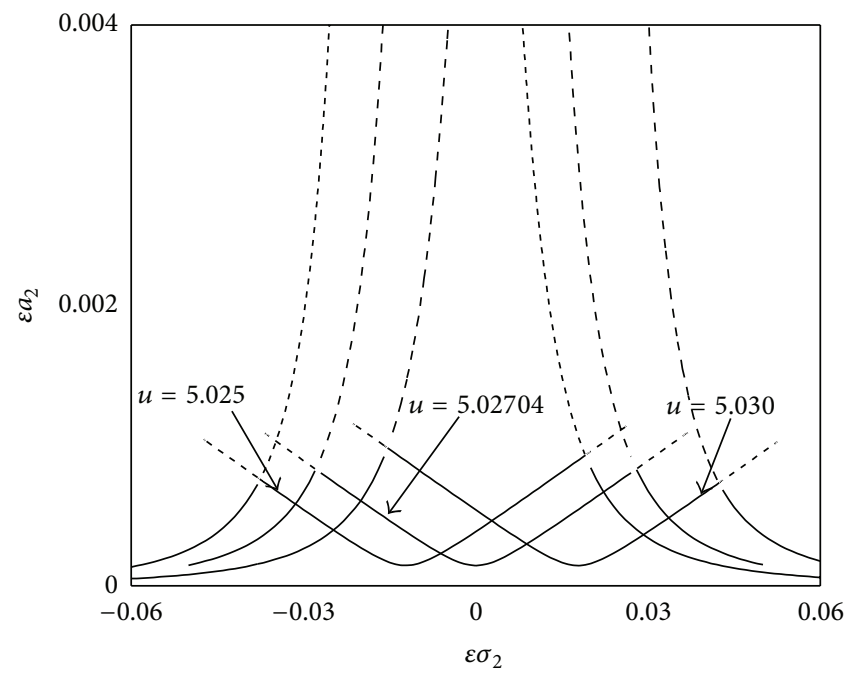

(b)

FIGURE 9: Amplitude-frequency curves of the second superharmonic resonance for different flow speeds: (a) the first mode and (b) the second mode.

two-mode responses become unsymmetrical in the near but not exact internal resonance $\left(\sigma_{1} \neq 0\right)$.

In the second superharmonic resonance with the $2: 1$ internal resonance, the pipe vibration becomes phenomenal abruptly when the excitation frequency is enough close to a half of the second natural frequency and even tends to be larger when the excitation frequency equals a half of the second natural frequency.

4.3. Summation Resonance: Steady-State Responses and Their Stability. For the study of the summation resonance of the first two modes (i.e., $\omega \approx \omega_{1}+\omega_{2}$ ) in presence of $2: 1$ internal resonance, the parameters are chosen as $M_{r}=0.447$, $P=-5, u=5.02704, \alpha=0.001, f=0.1$, and $\kappa=4$ unless other values are assigned.

Figure 10 illustrates the amplitude-frequency response curve of the summation resonance for different viscosity coefficients ( $\alpha=0.001,0.002$, and 0.003). As shown in Figure 10, in both modes, the amplitude-frequency curve consists of stable upper branches and unstable lower ones, and the amplitude of both branches decreases with the viscosity coefficients. However, there are no longer jumping 


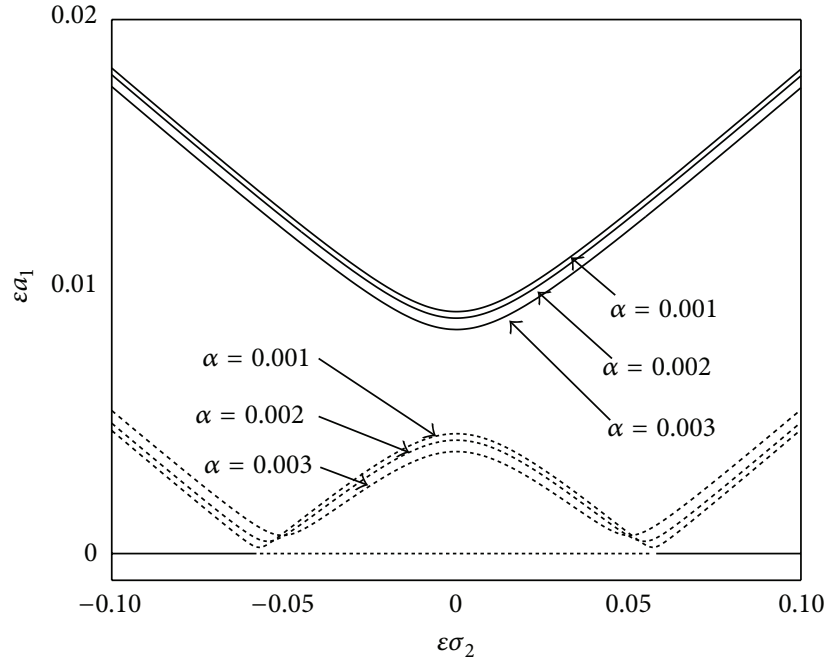

(a)

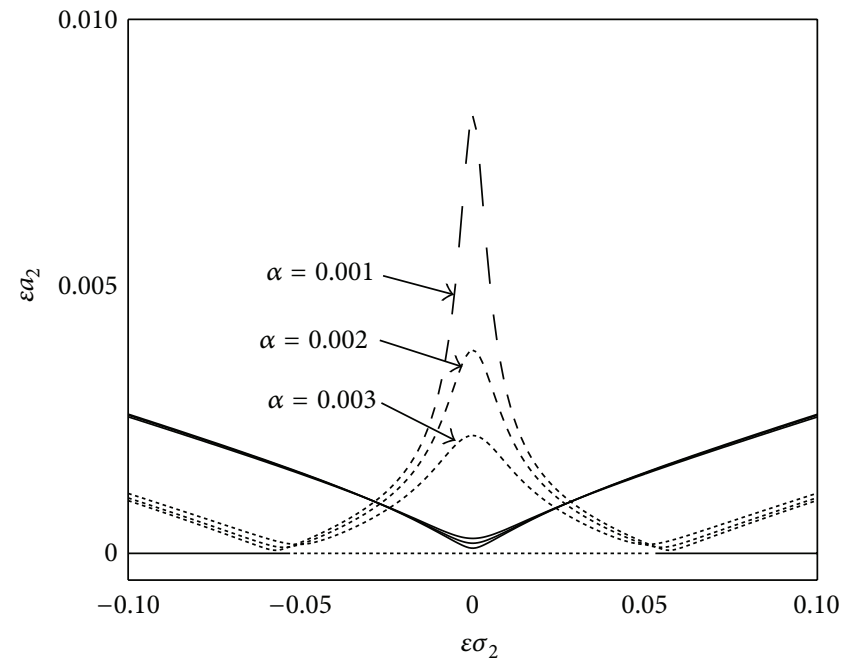

(b)

FIGURE 10: Amplitude-frequency curves of the summation resonance for different viscosity coefficients: (a) the first mode and (b) the second mode.

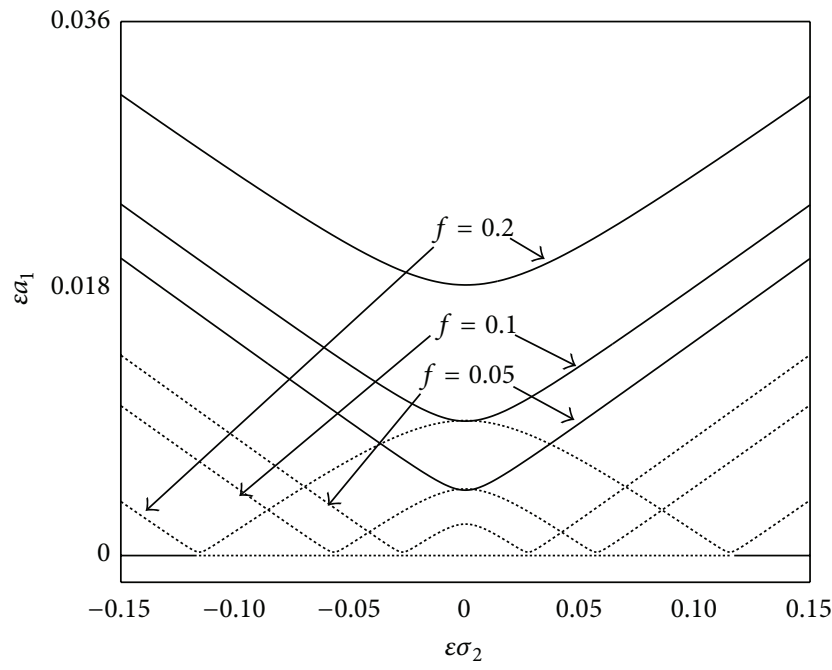

(a)

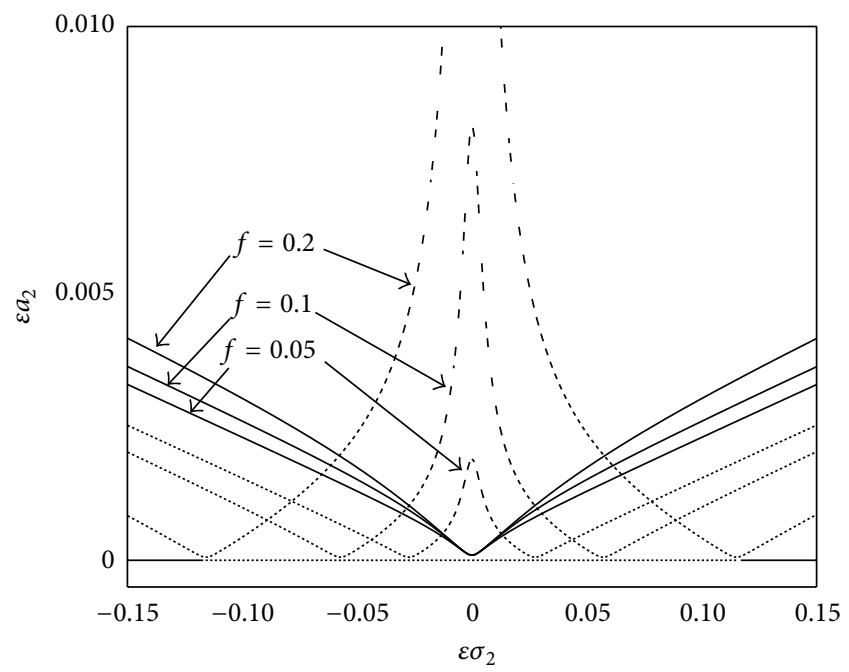

(b)

FIGURE 11: Amplitude-frequency curves of the summation resonance for different external excitation amplitudes: (a) the first mode and (b) the second mode.

phenomena. Besides, the amplitude of the stable response in the first mode is much larger than that in the second mode.

Figure 11 depicts the amplitude-frequency response curve of the summation resonance for different external excitation amplitudes $(f=0.05,0.1$, and 0.2$)$. As shown in Figure 11, the amplitudes of both stable and unstable responses increase with the external excitation amplitudes. As the excitation amplitudes here are much larger than those in the first subharmonic and the second superharmonic resonances calculated in Sections 4.1 and 4.2, the responses in the summation responses are much smaller than those in the subharmonic and the superharmonic resonances for the same parameters.

Figure 12 shows the amplitude-frequency response curves of the summation resonance for different nonlinearity coefficients $(\kappa=2,4$, and 6). As shown in Figure 12, the amplitudes of the stable responses decrease with the nonlinearity coefficients, and the trends reverse for the unstable responses when the detuning parameter is small enough.

Figure 13 demonstrates the amplitude-frequency response curves of the summation resonances for different flow 


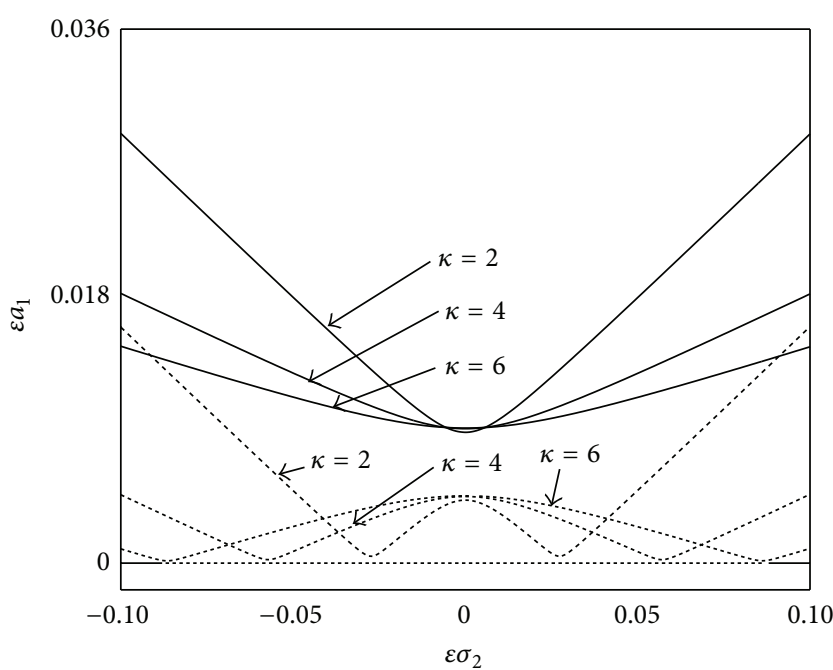

(a)

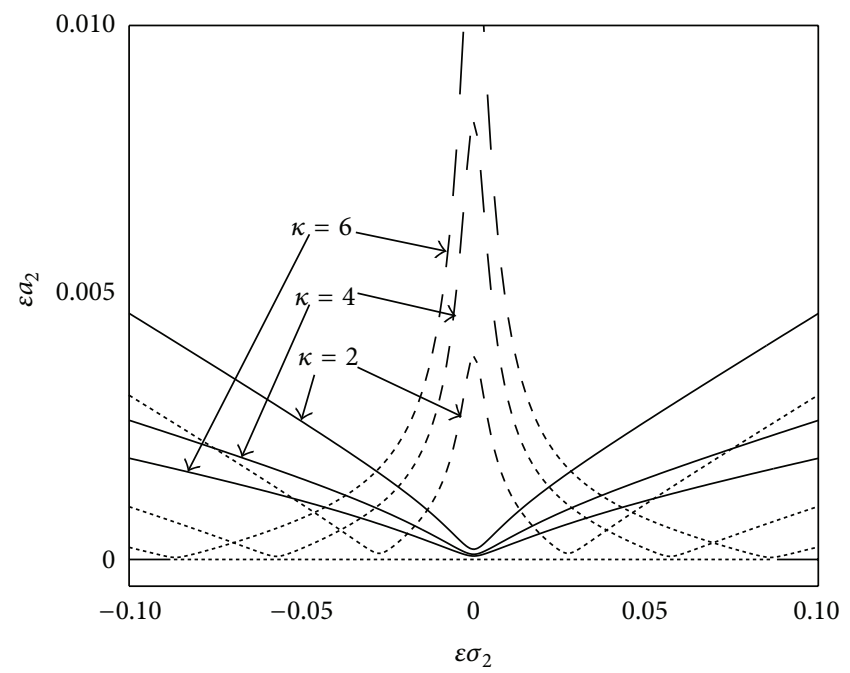

(b)

FIGURE 12: Amplitude-frequency curves of the summation resonance for different nonlinearity: (a) the first-mode and (b) the second-mode coefficients.

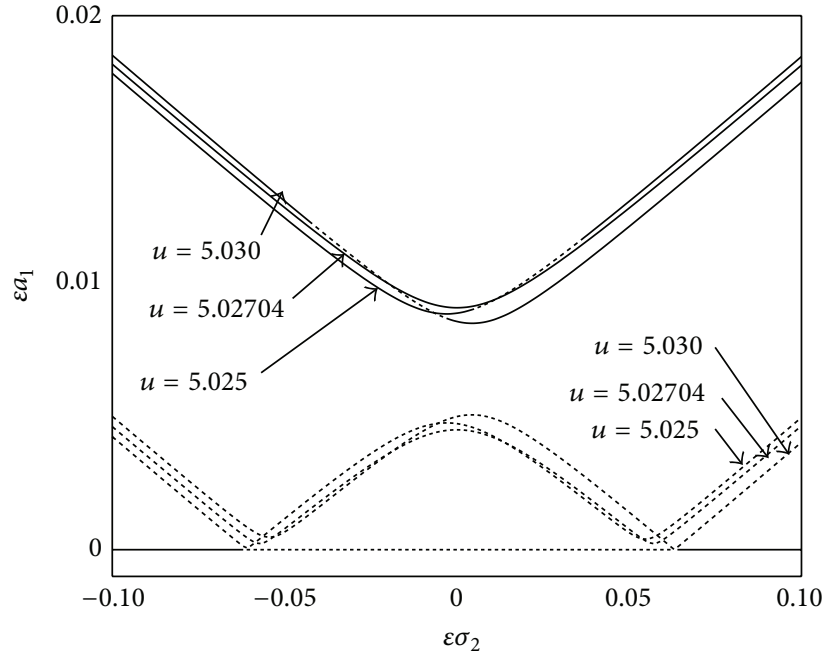

(a)

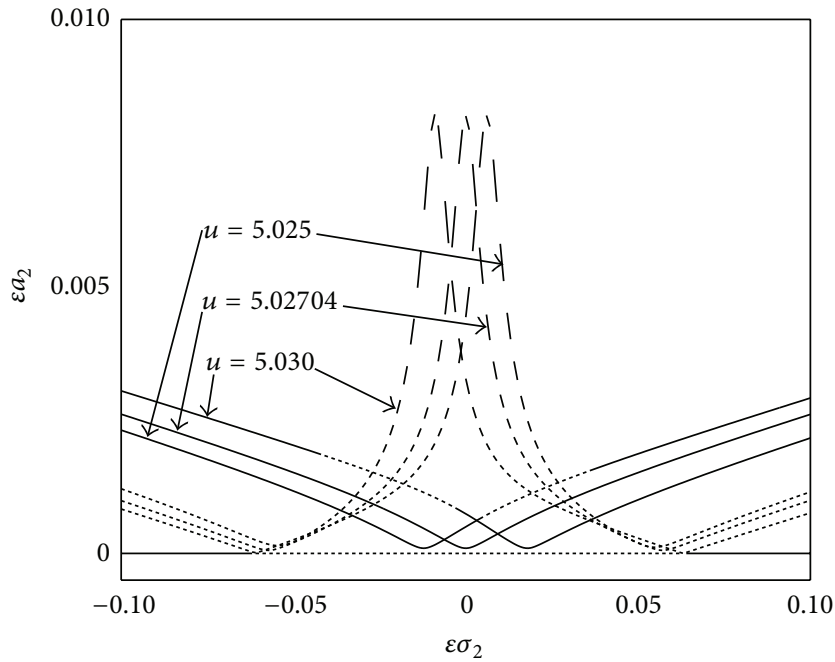

(b)

FIGURE 13: Amplitude-frequency curves of the summation resonance for different flow speeds: (a) the first mode and (b) the second mode.

speeds $(u=5.025,5.02704$, and 5.030). As shown in Figure 13, both the stable and the unstable responses become unsymmetrical in the near but not exact internal resonance $\left(\sigma_{1} \neq 0\right)$.

4.4. Difference Resonance: Steady-State Responses and Their Stability. To investigate the difference resonance of the first two modes (i.e., $\omega \approx \omega_{2}-\omega_{1}$ ) in presence of the $2: 1$ internal resonance, the parameters are chosen as $M_{r}=0.447, P=-5$, $u=5.02704, \alpha=0.001, f=0.001$, and $\kappa=4$ unless other values are assigned.

Figure 14 shows the amplitude-frequency response curves of the difference resonances for different viscosity coefficients ( $\alpha=0.001,0.002$, and 0.003 ). As shown in Figure 14, for small viscosity coefficients, the amplitude-frequency curves is with two types of jumping separated by a high no-jumping peak in the first mode and connected at a low position in the second mode. Even excluding the no-jumping peak, the amplitude of the first-mode response is much larger than that of the second-mode one.

Figure 15 demonstrates the amplitude-frequency response curves of the difference resonances for different external excitation amplitudes ( $f=0.0005,0.001$, and 0.002). As shown in Figure 15, the response amplitudes in both modes increase with the increasing amplitudes of external excitation. That is, the larger excitation amplitude leads to the higher jumping.

Figure 16 depicts the amplitude-frequency response curve of the difference resonances for different nonlinearity 


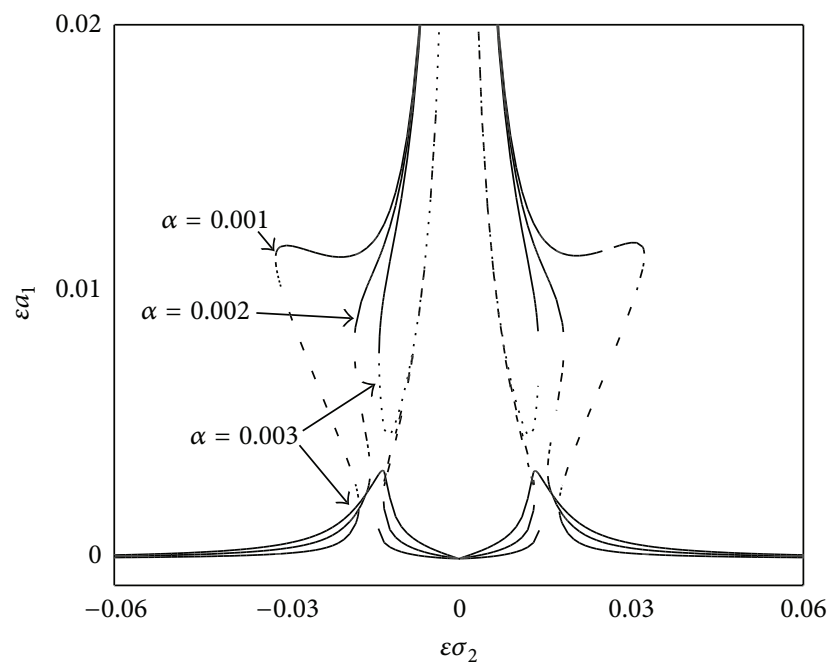

(a)

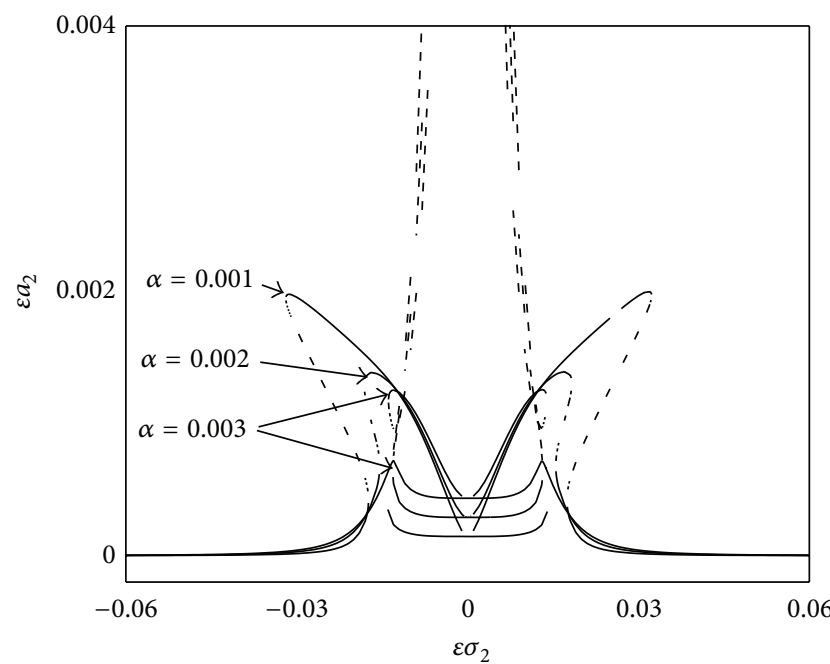

(b)

FIGURE 14: Amplitude-frequency curves of the difference resonance for different viscosity coefficients: (a) the first mode and (b) the second mode.

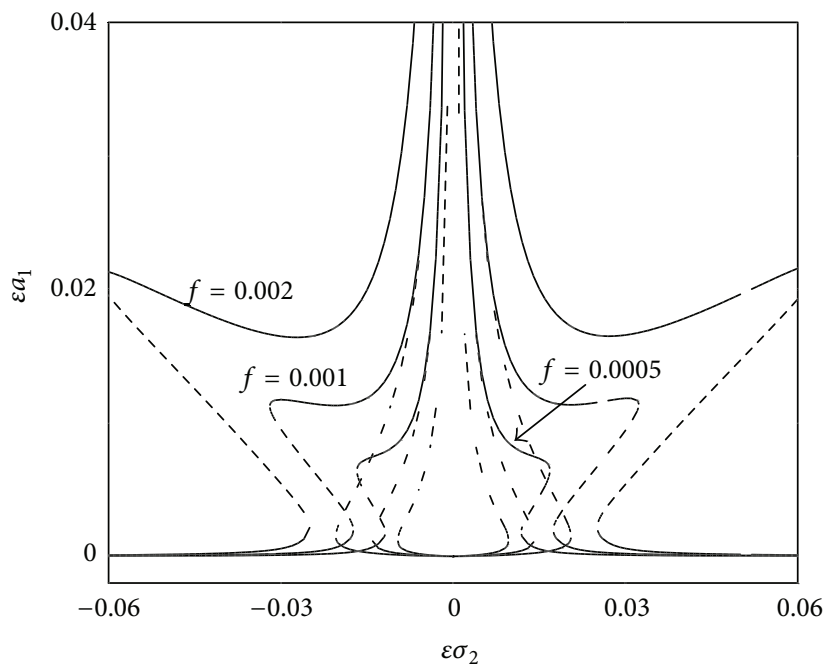

(a)

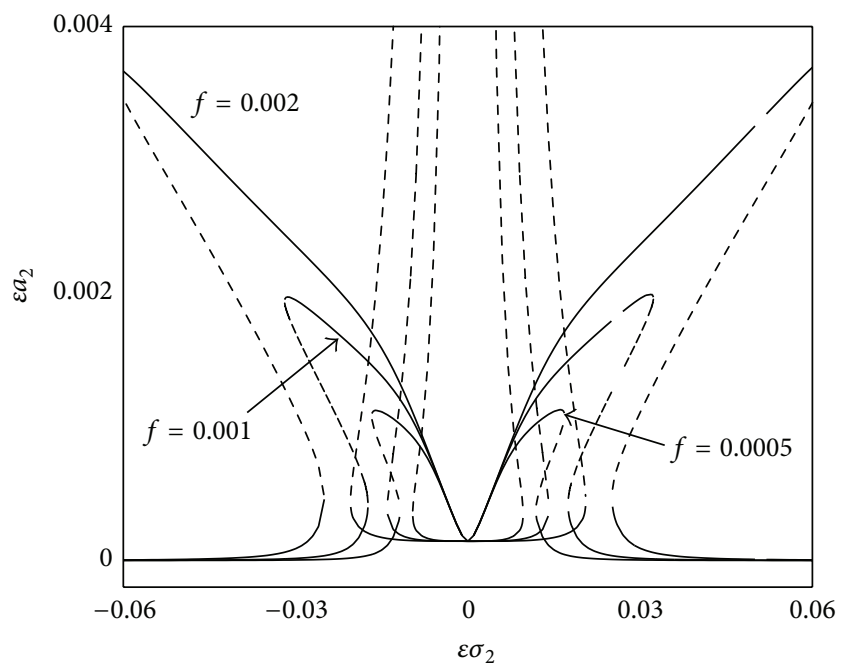

(b)

FIGURE 15: Amplitude-frequency curves of the difference resonance for different external excitation amplitudes: (a) the first mode and (b) the second mode.

coefficients $(\kappa=2,4$, and 6$)$. As shown in Figure 16 the response amplitudes in both modes decrease with the nonlinearity coefficients.

Figure 17 shows the amplitude-frequency response curves of the second superharmonic resonant for different flow speeds $(u=5.025,5.02704$, and 5.030). As shown in Figure 17, the amplitude-frequency curves in both modes become unsymmetrical in the near but not exact internal resonance $\left(\sigma_{1} \neq 0\right)$.

In the difference resonance of the first two modes with the presence of the $2: 1$ internal resonance, the first modal response, which dominates the pipe vibration, behaves similarly to the case of the second subharmonic resonance with the $2: 1$ internal resonance. It is physically understandable. As $\omega \approx \omega_{2}-\omega_{1}$ and $\omega_{2} \approx 2 \omega_{1}$, then $2 \omega \approx \omega_{2}$.

\section{Comparisons with Types of Numerical Integration}

Equations (29) for the first subharmonic resonance, (38) for the second superharmonic resonance, (46) for the sum resonance, and (53) for the difference resonance can be numerically integrated via the Runge-Kutta algorithm. For a set of parameters and the suitable initial conditions, each stable fixed point can be numerically calculated. The parameters are chosen as $M_{r}=0.447, P=-5, u=5.02704, \alpha=0.001$, 


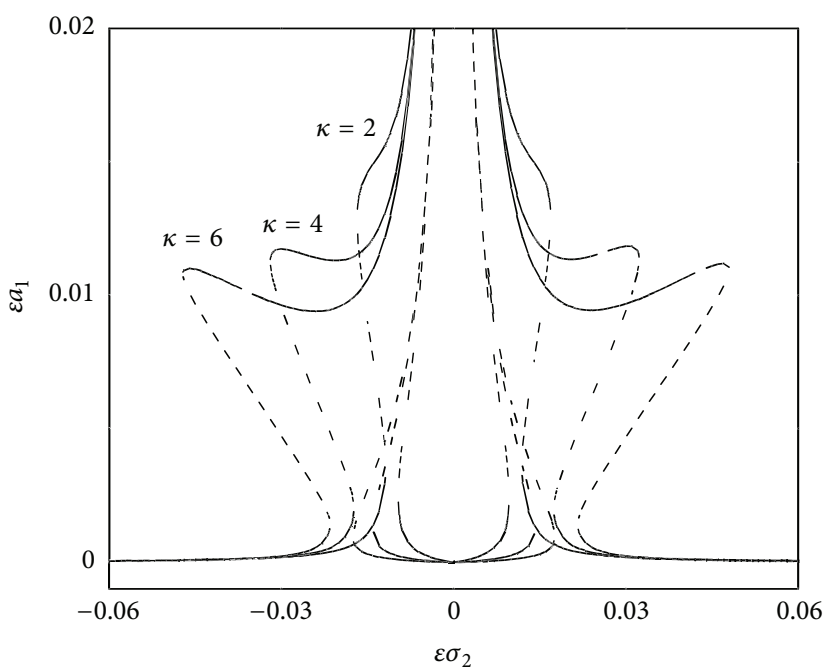

(a)

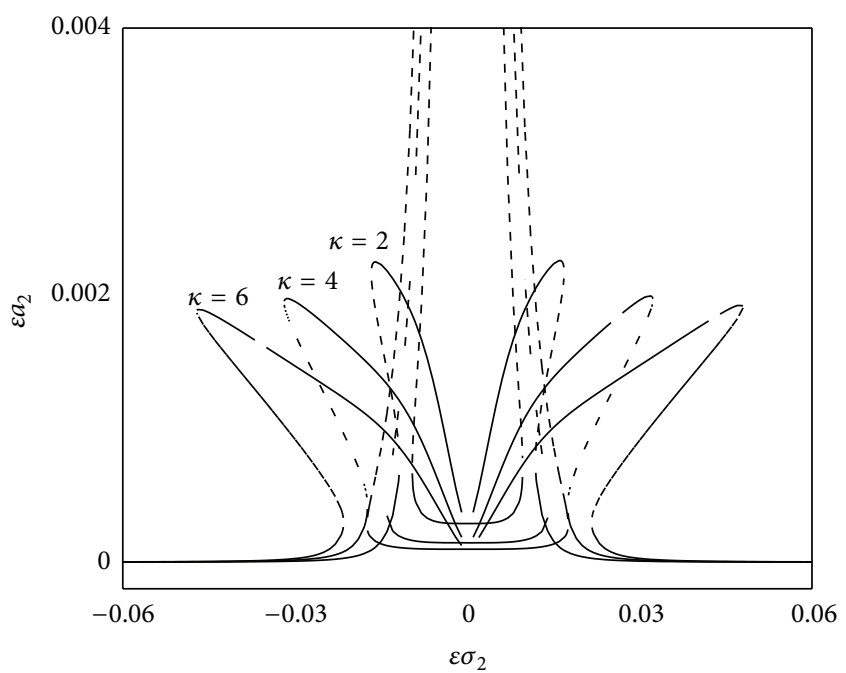

(b)

FIGURE 16: Amplitude-frequency curves of the summation resonance for different nonlinearity: (a) the first-mode and (b) the second-mode coefficients.

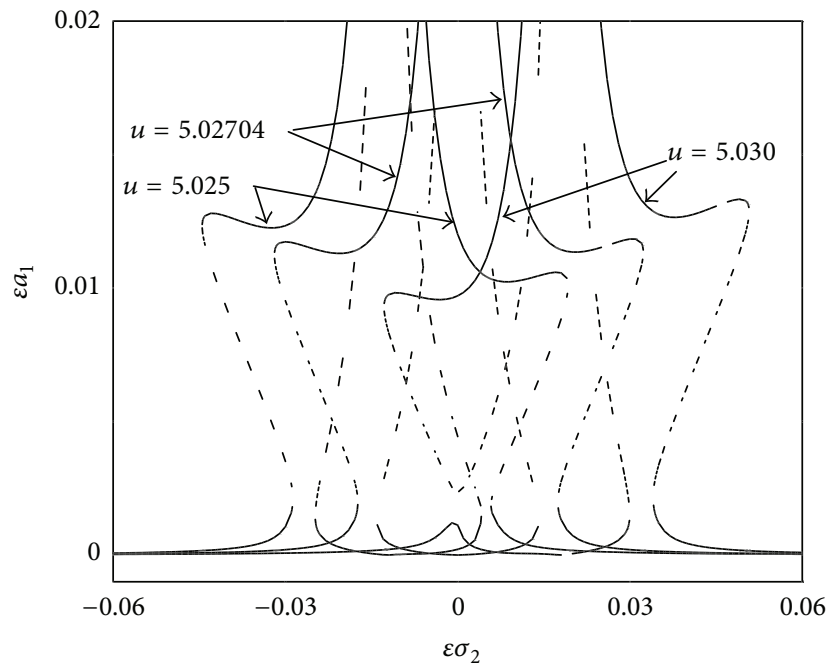

(a)

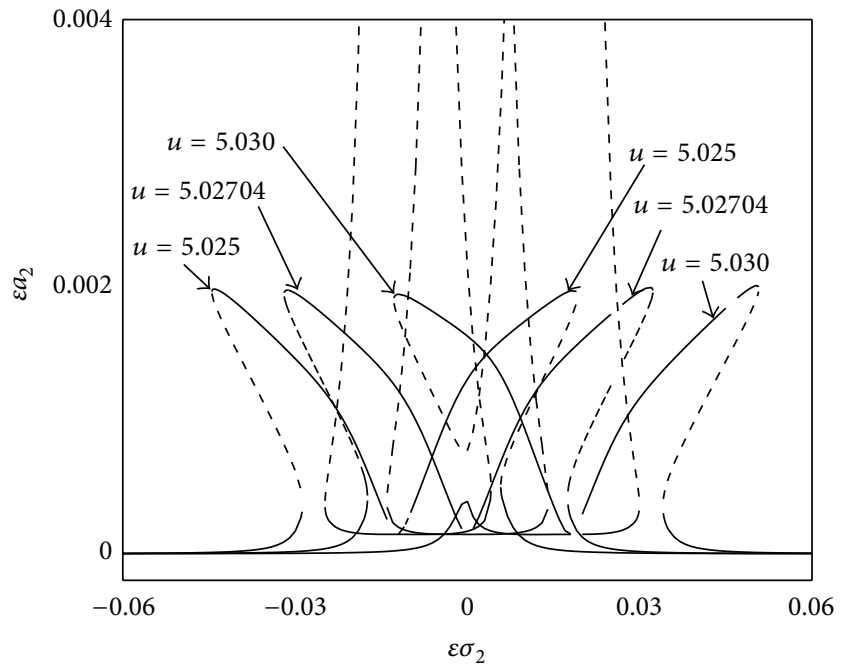

(b)

FIGURE 17: Amplitude-frequency curves of the difference resonance for different flow speeds: (a) the first mode and (b) the second mode.

$f=0.001$ (in the first subharmonic resonance, the second superharmonic resonance, and difference resonance), 0.1 (in the summation resonances), and $\kappa=4$. The stable parts of the amplitude-frequency response curves can be numerically determined by the integration for the varying excitation frequency. Numerical results are depicted in Figures 18-21 as solid dots for the subharmonic, the superharmonic, the summation, and the difference resonances, and analytical results are also depicted in Figures 18-21 as solid lines for the stable parts and dotted lines for the unstable parts for the sake of the comparisons. For stable steady-state responses, the analytical predictions and the numerical integration are in good agreement.

\section{Conclusions}

This paper is devoted to the investigation on steady-state responses of fluid-convey pipes to strong external harmonic excitations. The fluid flows in the speed larger than the critical one, and curved equilibriums are bifurcated. The motion under the investigation is around the curved equilibrium configuration. The governing equation is a nonlinear varyingcoefficient integro-partial-differential equation. The Galerkin method is applied to truncate the equation into a set of nonlinear ordinary differential equations. The method of multiple scales is developed to construct the relationship between the steady-state responses amplitude and the external excitation 


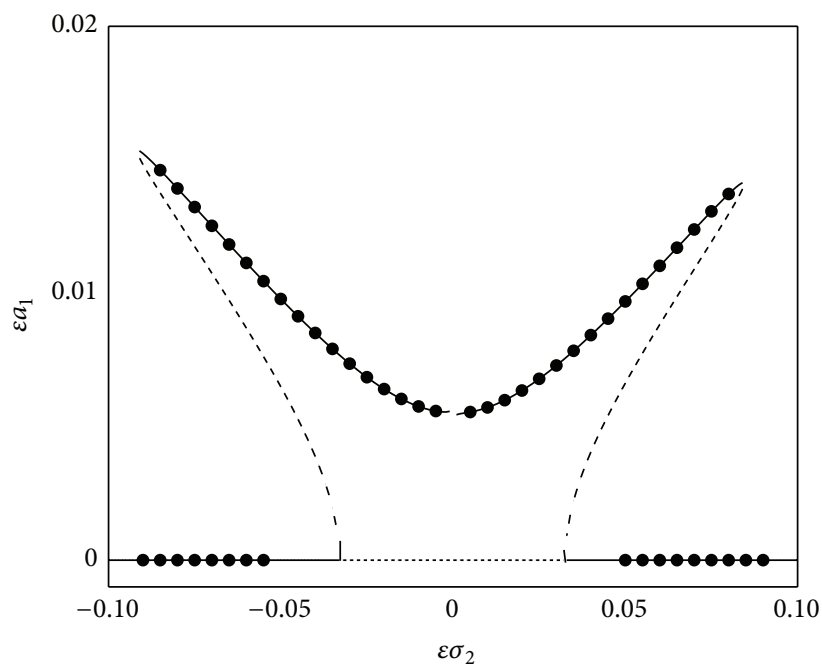

(a)

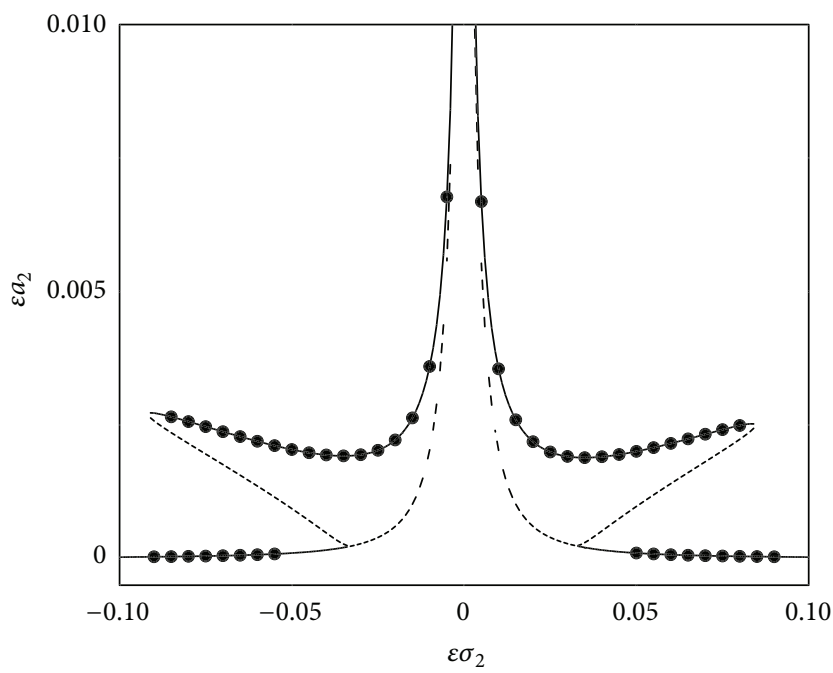

(b)

FIGURE 18: Comparisons in the first subharmonic resonance: (a) the first mode and (b) the second mode.

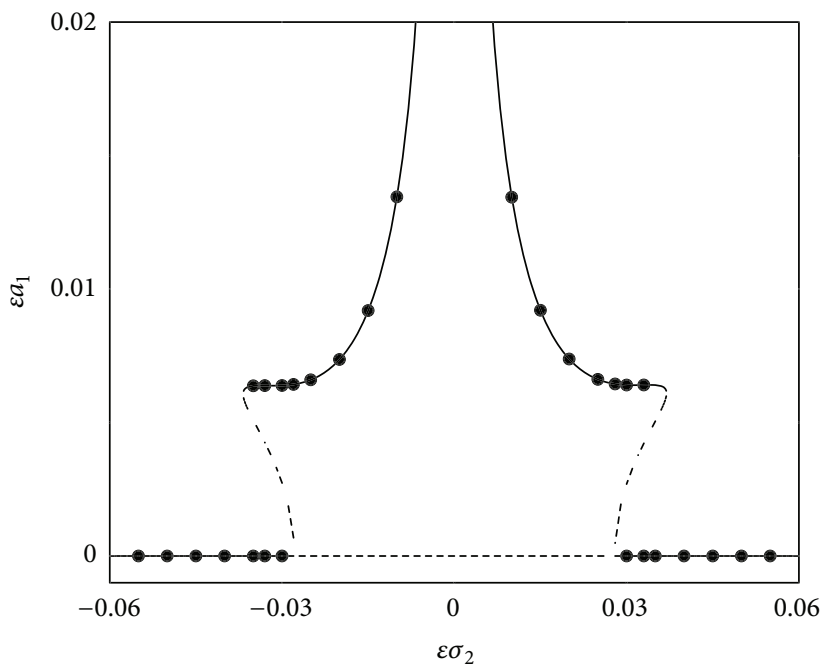

(a)

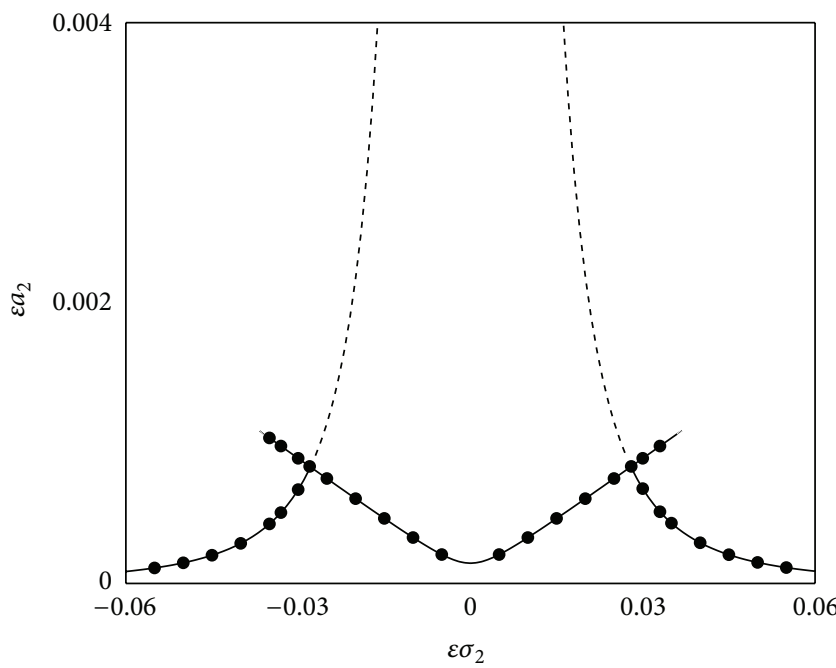

(b)

FIGURE 19: Comparisons in the second superharmonic resonance: (a) the first mode and (b) the second mode.

frequency in the external subharmonic, superharmonic combination resonances, respectively, in the present of the $2: 1$ internal resonance. Novel jumping phenomena are observed in the amplitude-frequency response curves. The analytical results are validated by comparing types of numerical integration. The investigation yields the following conclusions:

(1) In the supercritical flow speed regime, 2:1 internal resonance can be activated, while the 2:1 internal resonance does not occur in the subcritical speed case.

(2) In the presence of the 2:1 internal resonance, the amplitude-frequency curves possess two types of opposite-bending jumping connected to each other at the lowest point in the first subharmonic resonance of the first mode and the difference resonances; the amplitude-frequency curves possess two types of jumping separated by a high no-jumping peak in the first subharmonic resonance of the second mode, the second superharmonic resonance of the first modes, and the difference resonances of the first mode.

(3) Under the supercritical conditions, the steady-state vibration was analyzed for variations of the amplitude and oscillating frequency of the external excitation. Exciting the pipe in the first mode of vibration was found to be responsible of transferring energy from the shaker to the fluid, whereas higher modes of vibration played the role of transporting fluid with pipe vibrations of smaller amplitude. The amplitude 


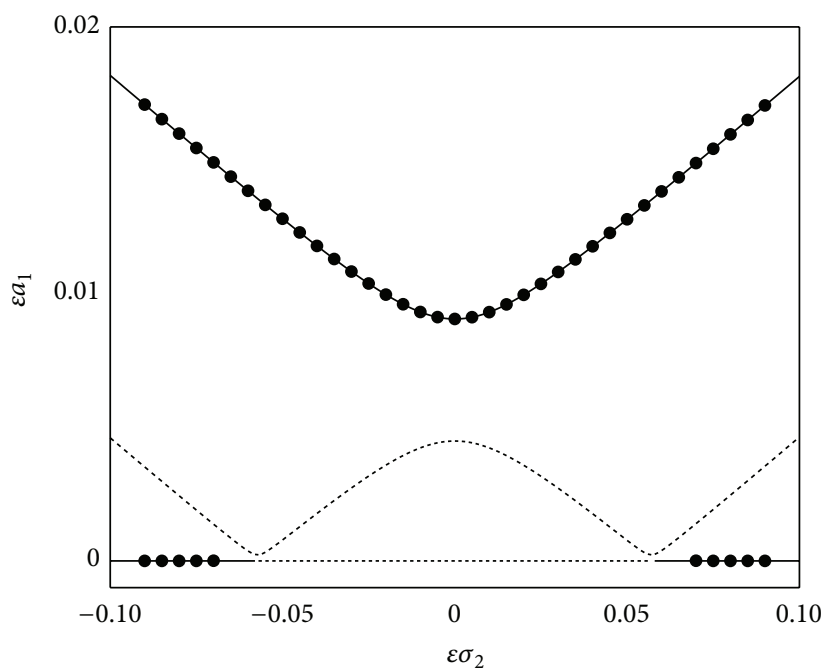

(a)

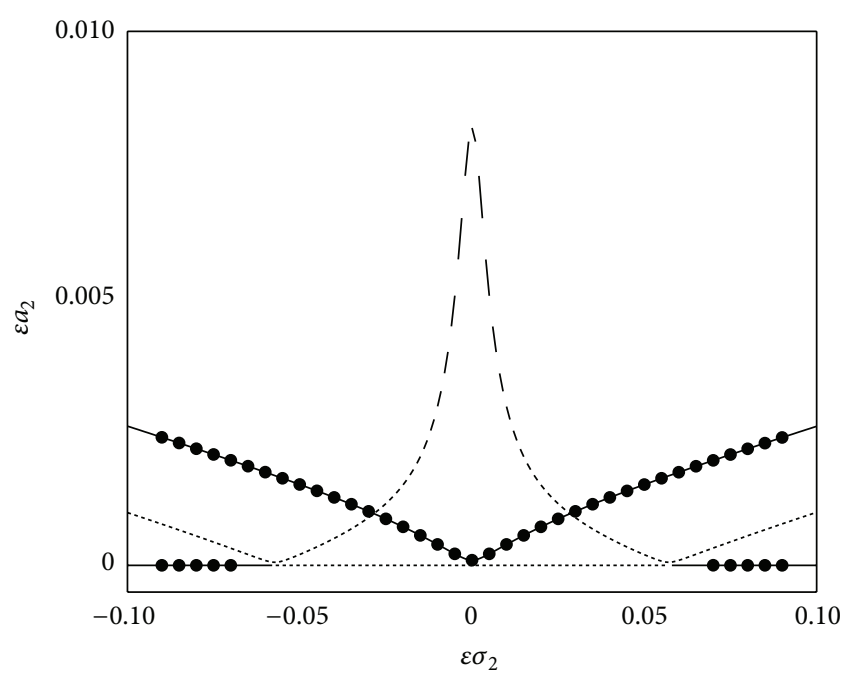

(b)

FIGURE 20: Comparisons in the summation resonance: (a) the first mode and (b) the second mode.

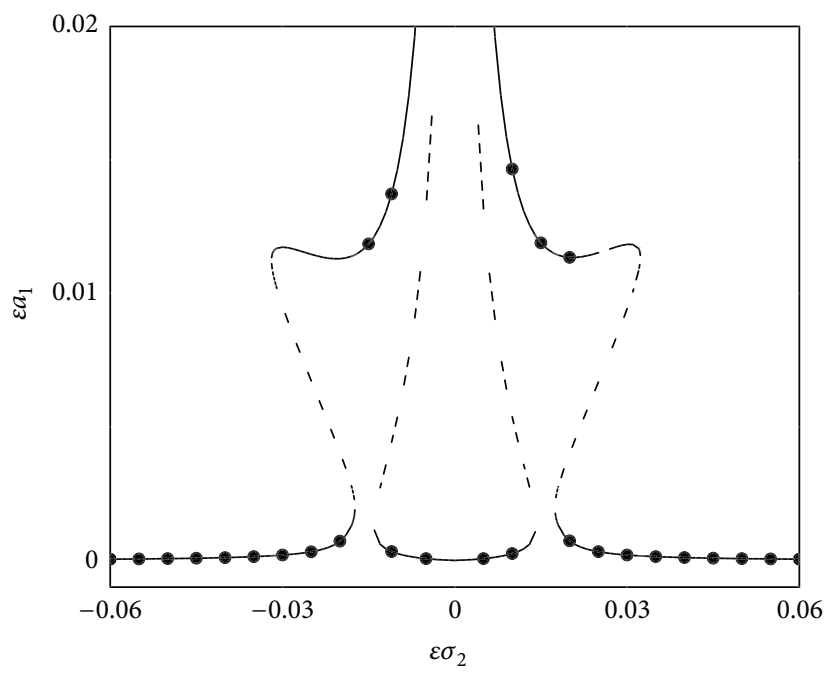

(a)

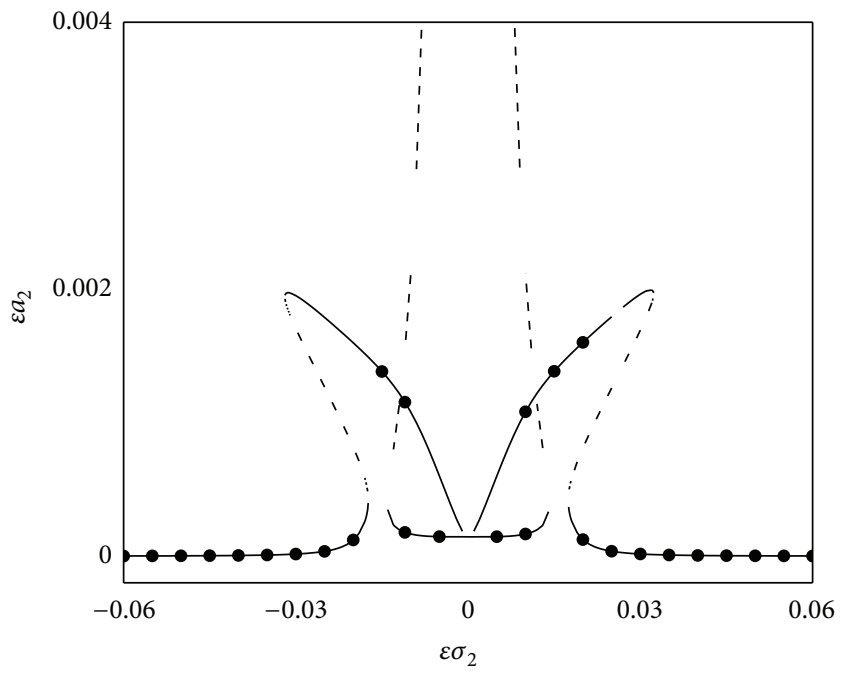

(b)

FIGURE 21: Comparisons in the difference resonance: (a) the first mode and (b) the second mode.

of the first-mode response is much larger than that of the second-mode one. The responses in the summation resonances are much smaller than those in the subharmonic, the superharmonic, and the difference resonances.

(4) Depending on the order of the nonlinearity such frequency relationships can cause the corresponding modes to be strongly coupled, and internal response is said to exist. The frequency response curve becomes multivalued and instabilities occur. The response can jump from one point to another on the frequency response curve in the unstable region. The response amplitude increases with the external excitation amplitude and decreases with the viscosity coefficient and the nonlinearity coefficient.
(5) The amplitude-frequency response curves become unsymmetrical in the near but not exact internal resonance.

\section{Conflict of Interests}

The authors declare that there is no conflict of interests regarding the publication of this paper.

\section{Acknowledgments}

This work was supported by the State Key Program of National Natural Science of China (no. 11232009), the National Natural Science Foundation of China (nos. 11302122, 11202136), Shanghai Leading Academic Discipline Project 
(no. S30106), the School Fund of Shanghai Second Polytechnic University under Grant no. EGD13XQD18, the Funding Projects for Teachers in the Universities of Shanghai under Grant no. ZZEGD13011, the Shanghai Leading Academic Discipline Project of Shanghai Second Polytechnic University (nos. XXKPY1305, XXKPY1311).

\section{References}

[1] M. P. Païdoussis, Fluid-Structure Interactions: Slender Structures and Axial Flow, vol. 1, Academic Press, London, UK, 1998.

[2] M. P. Païdoussis, Fluid-Structure Interactions: Slender Structures and Axial Flow, vol. 2, Academic Press, London, UK, 2004.

[3] R. A. Ibrahim, "Overview of mechanics of pipes conveying fluids. Part I. Fundamental studies," Journal of Pressure Vessel Technology, vol. 132, no. 3, Article ID 0340011, 32 pages, 2010.

[4] R. A. Ibrahim, "Mechanics of pipes conveying fluids-part II: applications and fluidelastic problems," Journal of Pressure Vessel Technology, vol. 133, no. 2, Article ID 024001, 2011.

[5] A. L. Thurman and C. D. Mote, "Nonlinear oscillation of a cylinder containing a flowing fluid," ASME Journal of Engineering for Industry, vol. 91, no. 4, pp. 1147-1155, 1969.

[6] P. J. Holmes, "Bifurcations to divergence and flutter in flowinduced oscillations: a finite dimensional analysis," Journal of Sound and Vibration, vol. 53, no. 4, pp. 471-503, 1977.

[7] M. H. Ghayesh and M. P. Païdoussis, "Three-dimensional dynamics of a cantilevered pipe conveying fluid, additionally supported by an intermediate spring array," International Journal of Non-Linear Mechanics, vol. 45, no. 5, pp. 507-524, 2010.

[8] M. H. Ghayesh, M. P. Païdoussis, and Y. Modarres-Sadeghi, "Three-dimensional dynamics of a fluid-conveying cantilevered pipe fitted with an additional spring-support and an end-mass," Journal of Sound and Vibration, vol. 330, no. 12, pp. 2869-2899, 2011.

[9] M. Nikolić and M. Rajković, "Bifurcations in nonlinear models of fluid-conveying pipes supported at both ends," Journal of Fluids and Structures, vol. 22, no. 2, pp. 173-195, 2006.

[10] R. H. Plaut, "Postbuckling and vibration of end-supported elastica pipes conveying fluid and columns under follower loads," Journal of Sound and Vibration, vol. 289, no. 1-2, pp. 264277, 2006.

[11] Y. Modarres-Sadeghi and M. P. Païdoussis, "Nonlinear dynamics of extensible fluid-conveying pipes, supported at both ends," Journal of Fluids and Structures, vol. 25, no. 3, pp. 535-543, 2009.

[12] Y.-L. Zhang and L.-Q. Chen, "Internal resonance of pipes conveying fluid in the supercritical regime," Nonlinear Dynamics, vol. 67 , no. 2, pp. 1505-1514, 2012.

[13] B. G. Sinir, "Pseudo-nonlinear dynamic analysis of buckled pipes," Journal of Fluids and Structures, vol. 37, pp. 151-170, 2013.

[14] Y.-L. Zhang and L.-Q. Chen, "Steady-state response of pipes conveying pulsating fluid near a 2:1 internal resonance in the supercritical regime," International Journal of Applied Mechanics, vol. 6, no. 5, Article ID 1450056, 2014.

[15] S. S. Chen, "Forced vibration of a cantilevered tube conveying fluid," The Journal of the Acoustical Society of America, vol. 48, no. 3, pp. 773-775, 1970.

[16] V. I. Gulyayev and E. Y. Tolbatov, "Forced and self-excited vibrations of pipes containing mobile boiling fluid clots," Journal of Sound and Vibration, vol. 257, no. 3, pp. 425-437, 2002.
[17] Y. S. Seo, W. B. Jeong, S. H. Jeong, J. S. Oh, and W. S. Yoo, "Finite element analysis of forced vibration for a pipe conveying harmonically pulsating fluid," JSME International Journal, Series C: Mechanical Systems, Machine Elements and Manufacturing, vol. 48, no. 4, pp. 688-694, 2006.

[18] F. Liang and B. Wen, "Forced vibrations with internal resonance of a pipe conveying fluid under external periodic excitation," Acta Mechanica Solida Sinica, vol. 24, no. 6, pp. 477-483, 2011.

[19] A. H. Nayfeh and B. Balachandran, "Modal interactions in dynamical and structural systems," ASME Applied Mechanics Review, vol. 42, no. 11S, pp. S175-S201, 1989.

[20] A. H. Nayfeh, Nonlinear Interactions: Analytical, Computational, and Experimental Methods, Wiley, New York, NY, USA, 1998.

[21] R. J. McDonald and N. Sri Namachchivaya, "Pipes conveying pulsating Fluid near a 0:1 resonance: local bifurcations," Journal of Fluids and Structures, vol. 21, no. 5-7, pp. 629-664, 2005.

[22] R. J. McDonald and N. Sri Namachchivaya, "Pipes conveying pulsating fluid near a 0:1 resonance: global bifurcations," Journal of Fluids and Structures, vol. 21, no. 5-7, pp. 665-687, 2005.

[23] J. Xu and Q.-B. Yang, "Flow-induced internal resonances and mode exchange in horizontal cantilevered pipe conveying fluid," Applied Mathematics and Mechanics, vol. 27, no. 7, pp. 819-832, 2006.

[24] L. N. Panda and R. C. Kar, "Nonlinear dynamics of a pipe conveying pulsating fluid with parametric and internal resonances," Nonlinear Dynamics, vol. 49, no. 1-2, pp. 9-30, 2007.

[25] L. N. Panda and R. C. Kar, "Nonlinear dynamics of a pipe conveying pulsating fluid with combination, principal parametric and internal resonances," Journal of Sound and Vibration, vol. 309, no. 3-5, pp. 375-406, 2008.

[26] M. H. Ghayesh, "Nonlinear forced dynamics of an axially moving viscoelastic beam with an internal resonance," International Journal of Mechanical Sciences, vol. 53, no. 11, pp. 1022-1037, 2011.

[27] M. H. Ghayesh, H. A. Kafiabad, and T. Reid, "Sub- and supercritical nonlinear dynamics of a harmonically excited axially moving beam," International Journal of Solids and Structures, vol. 49, no. 1, pp. 227-243, 2012.

[28] M. P. Païdoussis, S. P. Chan, and A. K. Misra, "Dynamics and stability of coaxial cylindrical shells containing flowing fluid," Journal of Sound and Vibration, vol. 97, no. 2, pp. 201-235, 1984.

[29] M. P. Païdoussis, A. K. Misra, and S. P. Chan, "Dynamics and stability of coaxial cylindrical shells conveying viscous fluid," Journal of Applied Mechanics-Transactions ASME, vol. 52, no. 2, pp. 389-396, 1985.

[30] M. P. Païdoussis, "Flow-induced instabilities of cylindrical structures," Applied Mechanics Reviews, vol. 40, no. 2, pp. 163175, 1987.

[31] J. A. Wickert, "Non-linear vibration of a traveling tensioned beam," International Journal of Non-Linear Mechanics, vol. 27, no. 3, pp. 503-517, 1992.

[32] A. H. Nayfeh and D. T. Mook, Nonlinear Oscillations, Wiley, New York, NY, USA, 1979. 


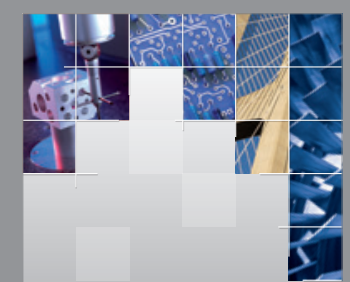

\section{Enfincering}
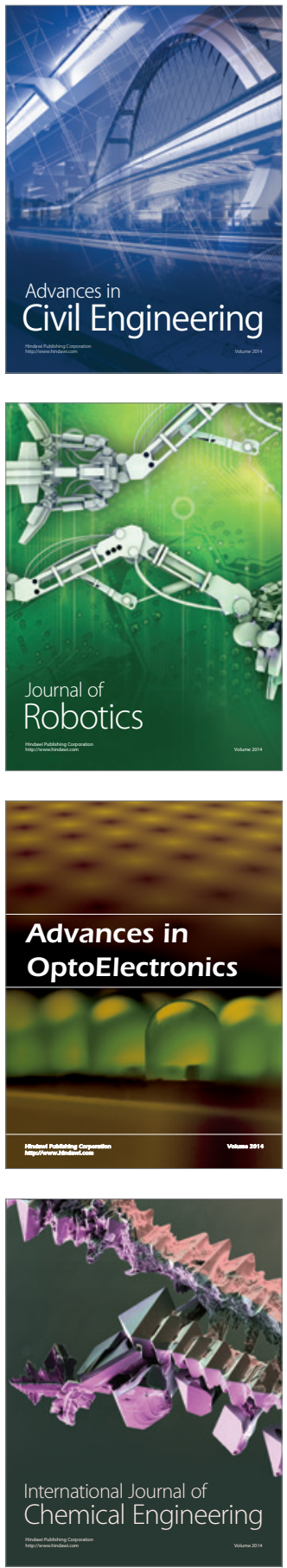

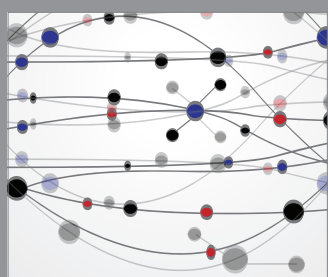

The Scientific World Journal

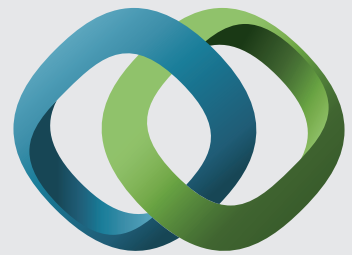

\section{Hindawi}

Submit your manuscripts at

http://www.hindawi.com
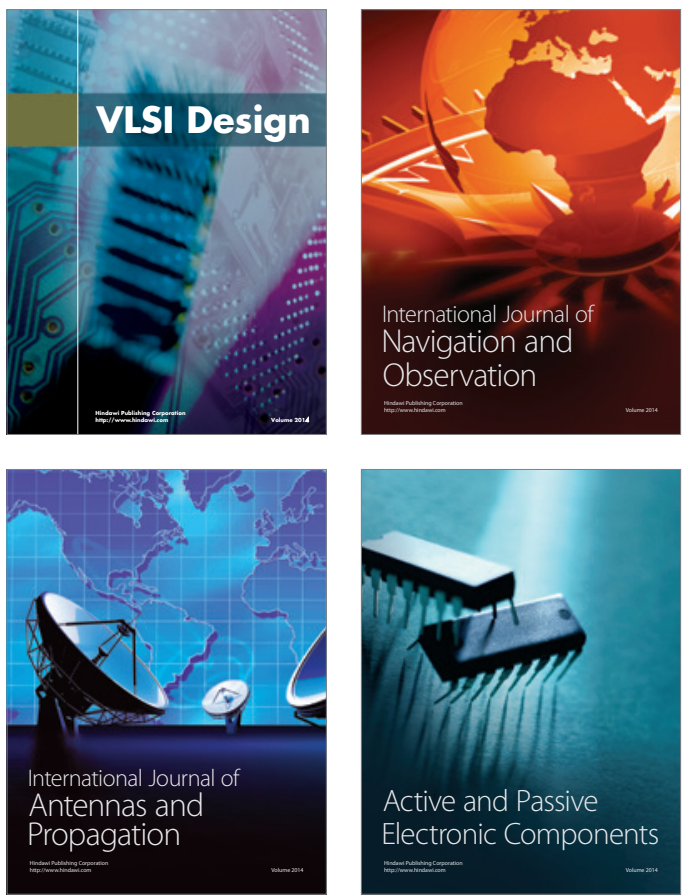
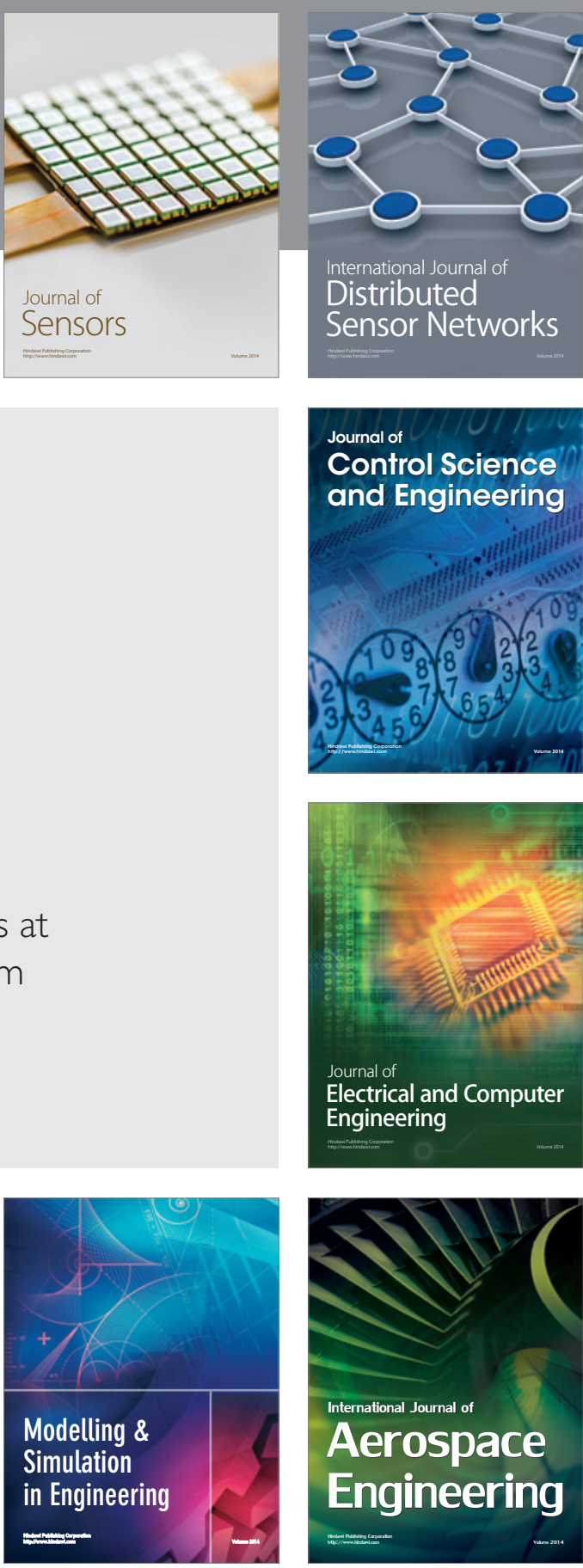

International Journal of

Distributed

Sensor Networks

Journal of

Control Science

and Engineering
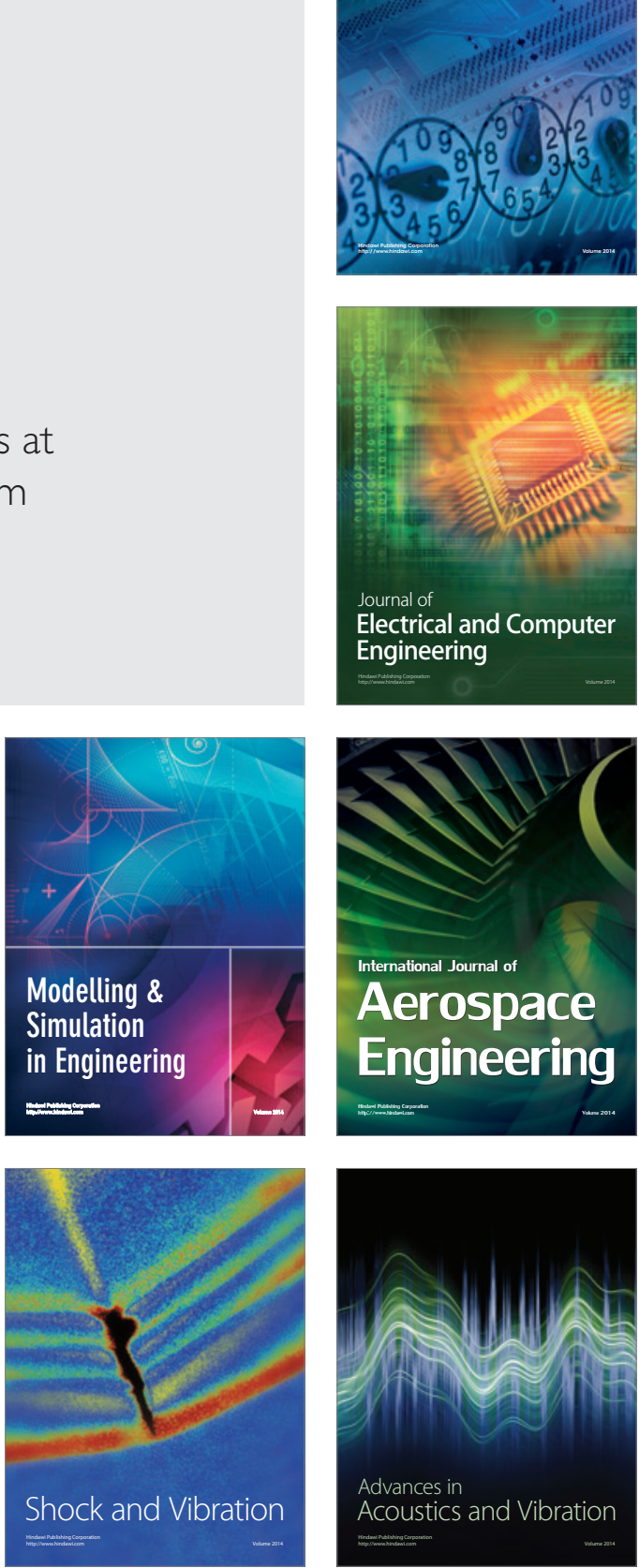